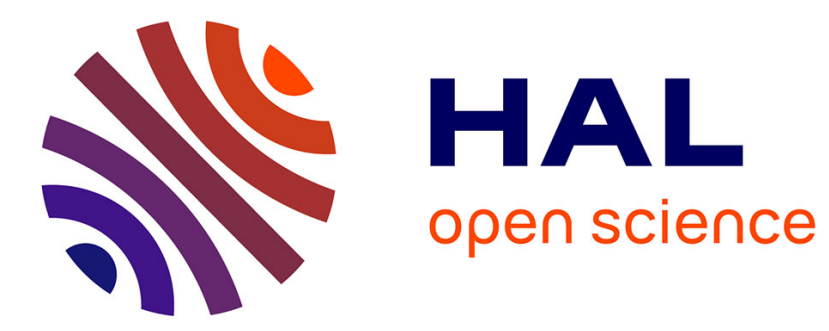

\title{
Design of fuzzy adaptive control for MIMO nonlinear time-delay systems with unknown actuator nonlinearities and control direction
}

\author{
Abdesselem Boulkroune, Mohammed M'Saad, Hassan Chekireb
}

\section{To cite this version:}

Abdesselem Boulkroune, Mohammed M'Saad, Hassan Chekireb. Design of fuzzy adaptive control for MIMO nonlinear time-delay systems with unknown actuator nonlinearities and control direction. Information Sciences, 2010, pp.5041-5059. 10.1016/j.ins.2010.08.034 hal-01059900

\author{
HAL Id: hal-01059900 \\ https://hal.science/hal-01059900
}

Submitted on 8 Sep 2014

HAL is a multi-disciplinary open access archive for the deposit and dissemination of scientific research documents, whether they are published or not. The documents may come from teaching and research institutions in France or abroad, or from public or private research centers.
L'archive ouverte pluridisciplinaire HAL, est destinée au dépôt et à la diffusion de documents scientifiques de niveau recherche, publiés ou non, émanant des établissements d'enseignement et de recherche français ou étrangers, des laboratoires publics ou privés. 


\title{
Design of a fuzzy adaptive controller for MIMO nonlinear time-delay systems with unknown actuator nonlinearities and unknown control direction
}

\author{
A. Boulkroune ${ }^{\mathrm{a}, *}$, M. M'Saad $^{\mathrm{b}}, \mathrm{H}$. Chekireb $^{\mathrm{c}}$ \\ a Department of Automatic, University of Jijel, Jijel 18000, Algeria \\ ${ }^{\mathrm{b}}$ GREYC, UMR 6072 CNRS, Université de Caen, ENSICAEN, 6 Bd Maréchal Juin, 14050 Caen Cedex, France \\ ${ }^{\mathrm{C}} \mathrm{LCP}$, Department of the Electric Engineering, ENP, Algiers, Algeria
}

Keywords:

MIMO nonlinear systems

Time-delay

Fuzzy control

Adaptive control

Dead-zone

Backlash

Nussbaum function

\begin{abstract}
A B S T R A C T
This paper aims at investigating the fuzzy adaptive control design for uncertain multivariable systems with unknown actuator nonlinearities and unknown control direction that possibly exhibit time-delay. The actuator nonlinearities involve dead-zone or backlash-like hysteresis, while the control direction is closely related to the sign of the control gain matrix. Two fuzzy adaptive controllers are proposed to deal with such an issue. The design of the first controller is mainly carried out in the free time-delay case, while the second control design is performed assuming that the system exhibits time-varying delays. Of practical interest, the adaptive compensation of the effects of the actuator nonlinearities requires neither the knowledge of their parameters nor the construction of their inverse. Furthermore, the lack of knowledge of the control direction is handled by incorporating in the control law a Nussbaum-type function. The effectiveness of the proposed fuzzy adaptive controllers is illustrated through simulation results.
\end{abstract}

\section{Introduction}

Thanks to the universal approximation theorem [56], a great deal of interest has been particularly dedicated to develop fuzzy adaptive control techniques to deal with the complex control problems involved in real world life systems, namely those concerning the multivariable nature, the time-delay and the actuator nonlinearities. In the following, one will provide a review of the available results bearing in mind the natural development of the fuzzy adaptive control research activity. This allows to emphasize the contributions of the paper with respect to the open literature.

The pioneering results on fuzzy adaptive control design to uncertain multivariable nonlinear systems are based on a suitable combination of a fuzzy adaptive controller and a robust compensator. The former allows to deal with system uncertainties, while the latter is mainly motivated by the ability to cope with those ubiquitous modelling approximation errors and external disturbances $[7,8,16,21,30,32,38,50-52]$. These contributions share the stability analysis nature and mainly differ from the involved robust compensator. The latter is particularly inspired by the supervisory control [16], sliding mode control $[8,21,30,38,51,52]$ and $H_{\infty}$ control $[7,16,32,50]$. There are two fundamental design issues that should to be pointed out. Firstly, how to deal with the singularity problem occurring when determining the inverse of the control gain matrix? Secondly, how to handle the prior knowledge requirement regarding the control gain matrix sign? The first issue has been

\footnotetext{
* Corresponding author.

E-mail addresses: boulkroune2002@yahoo.fr (A. Boulkroune), msaad@greyc.ensicaen.fr (M. M'Saad), chekireb@yahoo.fr (H. Chekireb).
} 
particularly investigated thanks to a suitable projection inside the parameter space up to an priori knowledge on the system under control, namely a feasible set in which the singularity problem does not happen [7,16,21,50,51]. A genuine procedure involving an appropriate regularization of the control gain matrix has been used in [30,52] up to an admissible tracking performance reduction. The second issue is more challenging. That is why, most fundamental results have been established assuming that the sign of the control gain matrix is known $[7,8,16,21,30,32,38,50-52]$. This is by no means a realistic assumption as pointed out in [61]. It is however worth noticing that such an issue has been investigated for specific class of multivariable nonlinear systems in [62,63] using the Nussbaum function concept introduced in [37]. The considered class of systems is relatively reduced to those that could be described by a lower triangular control structure with bounded highfrequency control gains.

Moreover several contributions have been made to properly deal with the actuator and sensor nonlinearities, namely dead-zones and hysteresis which are more a rule than an exception in the process control engineering. Besides the intrinsic performance limitations resulting from these nonlinearities, the control systems that are designed without taking into account the presence of these nonlinearities commonly exhibit bad performances or even unstable behaviour.

The dead-zone is a static memoryless nonlinearity which is commonly used to describe the insensitivity to small signals of a given component. The most straightforward way to cope with dead-zone nonlinearities consists in an achievable cancellation using their inverses. The seminal construction of a dead-zone adaptive inverse has been reported in $[46,47]$ where continuous-time and discrete-time adaptive dead-zone inverses for linear systems with unmeasurable dead-zone outputs were respectively built. Performance improvements have been obtained in $[2,14]$ where it was shown that an accurate adaptive cancellation of an unknown dead-zone can be asymptotically achieved provided that the dead-zone output is available for measurement. As the latter requirement is quite restrictive in the control process engineering, further investigations have been carried. Fuzzy compensators were proposed to deal with dead-zone in nonlinear industrial motion systems in $[25,27,31]$. Neural networks have been used to construct a dead-zone inverse compensator in [39]. An adaptive control system with an adaptive dead-zone inverse has been proposed in [57] up to a matching condition involving an ad hoc reference model. For a dead-zone with equal slopes, a robust adaptive control was developed in [58] for a class of nonlinear systems without constructing the inverse of the dead-zone. In [66], an adaptive output-feedback control using backstepping and smooth inverse function of the dead-zone was proposed for a class of nonlinear systems with unknown dead-zone. The underlying adaptive control system suffers however from the over-parameterization problem.

The non-differentiable and non-memoryless character of the hysteresis phenomenon is crucial from a control design point of view. The main issue consists in the lack of a suitable experimental modelling technique. This explains the variety of models that has been proposed to describe the hysteresis, namely the Ishlinskii hysteresis operator [29], the Duhem hysteresis operator [34] and the Preisach model [35]. It is however worth noticing that the most familiar and simple hysteresis model is the backlash described by two parallel lines connected via horizontal line segments. Several adaptive control systems have been proposed in $[1,44,48,49,67]$ to tackle backlash hysteresis using an achievable inverse. More recently, a robust adaptive output control of uncertain nonlinear systems with unknown but continuous backlash nonlinearity was proposed in [67]. This key feature consists in using a smooth adaptive inverse to compensate for the effect of the backlash within a backstepping control design framework. Of practical interest, the over-parameterization issue is handled using the concept of tuning functions. A robust variable structure controller has been proposed in [42] for systems involving a continuous backlash-like hysteresis without resorting to the hysteresis inverse. The robust variable structure controller fully exploits the solution properties of the differential equation describing the backlash hysteresis. Recent adaptive control schemes have been proposed in $[43,59,65]$ for nonlinear systems involving a continuous backlash-like hysteresis.

Finally, a remarkable research activity has been devoted to neural and fuzzy adaptive control for time-delay nonlinear systems. The corresponding contributions heavily borrow from the robust stability analysis approaches that have been developed for time-delay linear systems over the last years $[22,26,28,45]$. The seminal contributions have been devoted to the adaptive neural control for single-input single-output nonlinear time-delay systems in strict-feedback form $[18,19]$. Though the underlying control systems are intrinsically robust with respect the uncertainties resulting from unknown time-delays, they suffer from the applicability point of view. Indeed, some restrictions on the functions of delayed states have been introduced for technical considerations imposed by the adopted Lyapunov control design approach. More important contributions have made for multivariable nonlinear time-delay systems in block-triangular form using the adaptive neural control [20,62]. In [20], the neural networks are essentially used to handle the unknown functions in systems dynamics as well as the unknown bounds of the functions of delayed states. Unlike in $[18,19]$, the restrictive assumption on the function of delayed states has been removed thanks to a useful separation technique. The contribution [62] consists in designing an adaptive neural controller for nonlinear state time-varying delay systems in triangular control structure. The control design is particularly inspired from the fundamental developments in $[18,19]$ and shares hence the same limitations. A fuzzy adaptive controller, inspired from [18,19], has been recently proposed in [55] for nonlinear time-delay systems with unknown virtual control coefficients. Although an upper bound of time-varying delay functions is assumed to be known, only one parameter is tuned by the involved learning law as in [60].

Two fuzzy adaptive controllers are proposed in this paper for uncertain multivariable nonlinear systems with both unknown actuator nonlinearities and unknown sign of the control gain matrix. The design of the first controller is particularly carried out in the free time-delay case, while the second control design is performed assuming that the system exhibits timevarying delays. It is worth noting that the preliminary short versions of this first controller were presented as [5,6]. The main contributions of this paper with respect to the existing works in the literature are emphasized below. 
- The modelling assumptions have been relaxed with respect to those in [62,63], namely lower triangular control structure with bounded high-frequency control gains. These modelling requirements were mainly motivated by stability analysis and control design purposes.

- A unique Nussbaum-type function is used in order to estimate the true sign of the control gain matrix unlike in $[62,63]$ where many Nussbaum-type functions are used.

- The control gain matrix is appropriately decomposed in the product of a symmetric definite-positive matrix, a diagonal matrix with +1 or -1 on the diagonal and a unity upper triangular matrix. It is worth noticing that the diagonal matrix elements are nothing than the ratios of the signs of the leading principal minors of the control gain matrix. This matrix decomposition heavily borrows from [13,15,23,64].

- The stability analysis is relatively simple and different from that pursued in $[62,63]$. Recall that an integral Lyapunov function is considered in $[62,63]$ to avoid the controller singularity.

- Unlike in [62], the proposed controllers have three important features that are worth to be pointed. The first one is performance oriented, namely the tracking errors converge towards the origin. The second one is the singularity free control and the corresponding implementation feasibility. The third one is of practical interest as no prior knowledge on the timevarying delay is assumed. Notice that upper bounds on the time-delay and its derivative are commonly assumed to be known.

Throughout the paper, the notation $M^{T}$ denotes the transpose of a square matrix $M . \lambda_{\min }(M)$ and $\lambda_{\max }(M)$ denote the smallest and largest eigenvalues of this square matrix M. For $x \in R^{n},\|x\|=\left(x^{T} x\right)^{1 / 2}$ denotes the Euclidean norm of the vector.

\section{Problem statement and preliminaries}

Consider the following class of MIMO nonlinear time-delay systems described by:

$$
\left\{\begin{array}{l}
y_{1}^{\left(r_{1}\right)}=f_{1}(x)+h_{\tau 1}\left(x_{\tau}\right)+\sum_{j=1}^{p} g_{1 j}(x) N_{j}\left(v_{j}\right), \\
\vdots \\
y_{p}^{\left(r_{p}\right)}=f_{p}(x)+h_{\tau p}\left(x_{\tau}\right)+\sum_{j=1}^{p} g_{p j}(x) N_{j}\left(v_{j}\right), \\
x_{i}(t)=\phi_{x i}(t), \quad t \in\left[-\tau_{\max }, 0\right],
\end{array}\right.
$$

where $x=\left[x_{1}^{T}, \ldots, x_{p}^{T}\right]^{T} \in R^{r}$ is the overall state vector which is assumed available for measurement, where $x_{i}=$ $\left[y_{i}, \dot{y}_{i}, \ldots, y_{i}^{\left(r_{i}-1\right)}\right]^{T} \in R^{r_{i}}, \forall i=1, \ldots, p$ and $r_{1}+\cdots+r_{p}=r . x_{\tau}=\left[x_{1}^{T}\left(t-\tau_{1}(t)\right), \ldots, x_{p}^{T}\left(t-\tau_{p}(t)\right)\right]^{T} \in R^{r}$ is the delayed state vector, with $x_{\tau i}=x_{i}\left(t-\tau_{i}(t)\right)=\left[y_{i}\left(t-\tau_{i}(t)\right), \dot{y}_{i}\left(t-\tau_{i}(t)\right), \ldots, y_{i}^{\left(r_{i}-1\right)}\left(t-\tau_{i}(t)\right)\right]^{T} \in R^{r_{i}}, \forall i=1, \ldots, p, \tau_{1}(t), \ldots, \tau_{p}(t)$ are unknown timevarying delays. $\phi_{x 1}(t), \ldots, \phi_{x p}(t)$ are known continuous initial state vector functions, $\tau_{\max }=\max _{i}\left\{\tau_{i}^{*}\right\}$ with $\tau_{i}^{*}$ is an unknown positive constant which will be defined in Assumption 8. $v=\left[v_{1}, \ldots, v_{p}\right]^{T} \in R^{p}$ is the control input vector, $N_{i}\left(v_{i}\right)=u_{i}: R \rightarrow R$ is the actuator nonlinearity which is assumed here an unknown dead-zone or unknown backlash hysteresis. We consider $y=\left[y_{1}, \ldots, y_{p}\right]^{T} \in R^{p}$ as an output vector. $f_{i}(x), h_{\tau i}\left(x_{\tau}\right), i=1, \ldots, p$ are continuous unknown nonlinear functions, and $g_{i j}(x), i$, $j=1, \ldots, p$ are continuous unknown nonlinear $C^{1}$ functions.

Let us also denote

$$
\begin{aligned}
& y^{(r)}=\left[y_{1}^{\left(r_{1}\right)} \ldots y_{p}^{\left(r_{p}\right)}\right]^{T}, \quad F(x)=\left[f_{1}(x) \ldots f_{p}(x)\right]^{T}, \\
& H_{\tau}\left(x_{\tau}\right)=\left[h_{\tau 1}\left(x_{\tau}\right) \ldots h_{\tau p}\left(x_{\tau}\right)\right]^{T}, \quad G(x)=\left[\begin{array}{lll}
g_{11}(x) & \ldots & g_{1 p}(x) \\
\vdots & \ddots & \vdots \\
g_{p 1}(x) & \ldots & g_{p p}(x)
\end{array}\right] .
\end{aligned}
$$

Then, the system (1) can be rewritten in the following compact form:

$$
y^{(r)}=F(x)+G(x) u+H_{\tau}\left(x_{\tau}\right)
$$

where $u=\left[N_{1}\left(v_{1}\right), \ldots, N_{p}\left(v_{p}\right)\right]^{T}$ is the actuator output vector.

Assumption 1. The desired trajectory vector, $x_{d}=\left[x_{d 1}^{T}, \ldots, x_{d p}^{T}, y_{d 1}^{\left(r_{1}\right)}, \ldots, y_{d p}^{\left(r_{p}\right)}\right]^{T}$ with $x_{d i}=\left[y_{d i}, \dot{y}_{d i}, \ldots, y_{d i}^{\left(r_{i}-1\right)}\right]^{T}$ for $i=1, \ldots, p$, is supposed to be continuous, bounded and available for measurement. Then $x_{d} \in \Omega_{x_{d}} \subset R^{r+p}$ with $\Omega_{x_{d}}$ is a known compact set. 
The objective of this paper is to design a stable control system allowing the system output vector $y$ to follow a specified desired trajectory $y_{d}=\left[y_{d 1}, \ldots, y_{d p}\right]^{T} \in R^{p}$.

Let us define the tracking error as

$$
\begin{gathered}
e_{1}=y_{d 1}-y_{1}, \\
\vdots \\
e_{p}=y_{d p}-y_{p}
\end{gathered}
$$

and the filtered tracking error as

$$
S=\left[S_{1}, \ldots, S_{p}\right]^{T},
$$

with

$$
S_{i}=\left[\frac{d}{d t}+\lambda_{i}\right]^{r_{i}-1} e_{i}, \quad \text { for } \lambda_{i}>0, \quad \forall i=1, \ldots, p .
$$

Then, we can rewrite (5) as follows:

$$
S_{i}=\sum_{j=0}^{r_{i}-1}\left(\begin{array}{l}
r_{i}-1 \\
j
\end{array}\right) \lambda_{i}^{r_{i}-1-j} e_{i}^{(j)}=\lambda_{i}^{r_{i}-1} e_{i}+\left(r_{i}-1\right) \lambda_{i}^{r_{i}-2} \dot{e}_{i}+\cdots+\left(r_{i}-1\right) \lambda_{i} e_{i}^{\left(r_{i}-2\right)}+e_{i}^{\left(r_{i}-1\right)},
$$

with $i=1, \ldots, p$.

Notice that if we choose $\lambda_{i}>0$, with $i=1, \ldots, p$, then the roots of the polynomial $H_{i}(s)=\lambda_{i}^{r_{i}-1}+\left(r_{i}-1\right) \lambda_{i}^{r_{i}-2} s+\cdots+$ $\left(r_{i}-1\right) \lambda_{i} S^{r_{i}-2}+s^{r_{i}-1}$ related to the characteristic equation of $S_{i}=0$ are all in the open left-half plane.

The relation (6) can be rewritten in the following compact form

$$
S_{i}=C_{i}^{T} E_{i},
$$

with

$$
\begin{aligned}
E_{i} & =\left[e_{i} \dot{e}_{i} \ldots e_{i}^{\left(r_{i}-2\right)} e_{i}^{\left(r_{i}-1\right)}\right]^{T}, \\
C_{i}^{T} & =\left[\lambda_{i}^{r_{i}-1}\left(r_{i}-1\right) \lambda_{i}^{r_{i}-2} \ldots\left(r_{i}-1\right) \lambda_{i} 1\right] .
\end{aligned}
$$

Consequently, the vector $S$ takes the form:

$$
S=C^{T} E,
$$

where

$$
\begin{aligned}
& C^{T}=\operatorname{diag}\left[C_{1}^{T} C_{2}^{T} \ldots C_{p}^{T}\right]_{(p \times r)}, \\
& E=\left[E_{1}^{T} E_{2}^{T} \ldots E_{p}^{T}\right]_{(r \times 1)}^{T},
\end{aligned}
$$

From (8) and (9), the dynamic of $S_{i}$ is described by:

$$
\dot{S}_{i}=C_{i}^{T} \dot{E}_{i}=0 e_{i}+C_{i}^{T} \dot{E}_{i}=\left[0 \lambda_{i}^{r_{i}-1}\left(r_{i}-1\right) \lambda_{i}^{r_{i}-2} \ldots\left(r_{i}-1\right) \lambda_{i}\right] E_{i}+e_{i}^{\left(r_{i}\right)}=C_{r i}^{T} E_{i}+e_{i}^{\left(r_{i}\right)}, \quad \text { and } \quad i=1, \ldots, p,
$$

where

$$
C_{r i}^{T}=\left[0 \lambda_{i}^{r_{i}-1}\left(r_{i}-1\right) \lambda_{i}^{r_{i}-2} \ldots\left(r_{i}-1\right) \lambda_{i}\right] .
$$

The dynamic of $S$ can be rewritten as follows:

$$
\dot{S}=C_{r}^{T} E+e^{(r)}
$$

where

$$
\begin{aligned}
& C_{r}^{T}=\operatorname{diag}\left[C_{r 1}^{T} C_{r 2}^{T} \ldots C_{r p}^{T}\right]_{(p \times r)}, \\
& e^{(r)}=\left[e_{1}^{\left(r_{1}\right)} e_{2}^{\left(r_{2}\right)} \ldots e_{p}^{\left(r_{p}\right)}\right]^{T},
\end{aligned}
$$

with

$$
e^{(r)}=y_{d}^{(r)}-y^{(r)},
$$


where $y^{(r)}=\left[y_{1}^{\left(r_{1}\right)} y_{2}^{\left(r_{2}\right)} \cdots y_{p}^{\left(r_{p}\right)}\right]^{T}$ is previously defined, and

$$
y_{d}^{(r)}=\left[y_{d 1}^{\left(r_{1}\right)} y_{d 2}^{\left(r_{2}\right)} \cdots y_{d p}^{\left(r_{p}\right)}\right]^{T} \text {. }
$$

From (18), we can rewrite (15) as follows:

$$
\dot{S}=C_{r}^{T} E+y_{d}^{(r)}-y^{(r)} .
$$

Thereafter, (20) will be used in the development of the fuzzy controllers and the stability analysis.

\subsection{Actuator nonlinearities}

Based on the previous results $[5,6,42,43,58,59,65]$, we can describe the output of the actuator nonlinearity (dead-zone or backlash) as follows:

$$
u_{i}=N_{i}\left(v_{i}\right)=b_{i i} v_{i}+d_{i}\left(v_{i}\right), \quad \forall i=1, \ldots, p,
$$

where $b_{i i}$ is a positive parameter of the actuator nonlinearity, and $d_{i}\left(v_{i}\right)$ is a bounded function characterising the actuator nonlinearity. We have $\left|d_{i}\left(v_{i}\right)\right| \leqslant d_{i}^{*}$, with $d_{i}^{*}$ is a positive constant. For more details about the dead-zone and backlash models used here, the reader is referred to $[5,6,42,43,58,59,65]$.

\section{Assumption 2}

(a) The actuator output $u_{i}$ (i.e. $N_{i}\left(v_{i}\right)$ ) is not available for measurement.

(b) The bound $d_{i}^{*}$ and the parameter $b_{i i}$ are unknown. But, the sign of $b_{i i}$ is known.

Now, let us denote

$$
\begin{aligned}
& d(v)=\left[d_{1}\left(v_{1}\right), d_{2}\left(v_{2}\right), \ldots, d_{p}\left(v_{p}\right)\right]^{T}, \\
& d^{*}=\left[d_{1}^{*}, d_{2}^{*}, \ldots, d_{p}^{*}\right]^{T}, \\
& B=\operatorname{diag}\left[b_{11}, b_{22}, \ldots, b_{p p}\right] .
\end{aligned}
$$

Then, Eq. (21) can be rewritten as follows:

$$
u=B v+d(v),
$$

where $B$ is a diagonal positive-definite matrix, and $d(v)$ can be treated as bounded disturbances.

\subsection{Decomposition of the control gain matrix}

Motivated by Chen et al. [13], Costa et al. [15] and Hsu et al. [23], we will need the following useful lemma in the control design in Section 3.1.

Lemma 1 [15]. Any real matrix $G(x) \in R^{P \times P}$ with non-zero leading principal minors can be decomposed as follows:

$$
G(x)=G_{s}(x) D T(x)
$$

where $G_{s}(x) \in R^{P \times P}$ is a symmetric positive-definite matrix, $D \in R^{P \times P}$ is a diagonal matrix whose elements are +1 or -1 and $\mathrm{T}(\mathrm{x}) \in R^{P \times P}$ is a unity upper triangular matrix. The diagonal elements $d_{i i}$ of $D$ are nothing else than the ratios of the signs of the leading principal minors of $G(x)$.

\section{Proof of Lemma 1. See $[15,41]$.}

It is worth noting that the decomposition (23) of the matrix $G(x)$ is very useful. The symmetric positive-definite matrix $G_{s}(x)$ will be exploited in the stability analysis. The unity upper triangular matrix $T(x)$ allows for algebraic loop free sequential determination of control components. The diagonal elements $d_{i i}$ of the matrix $D$ are nothing than the ratios of the signs of the leading principal minors of $G(x)[5,6,13,15,23]$. This implies the following facts when the control gain matrix has nonzero leading principal minors:

- If $G(x)$ is positive-definite, then $D=I_{P}$,

- If $G(x)$ is negative-definite, then $D=-I_{p}$, and

- If $G(x)$ is indefinite, the diagonal elements of the matrix $D$ are +1 and -1 . 
Now, we propose a second lemma that is inspired from Lemma 1 and that will be used in the controller design in Section 3.2.

Lemma 2. For Any real matrix $G(x) \in R^{P \times P}$ with non-zero leading principal minors and any real constant diagonal positive-definite matrix $B=\operatorname{diag}\left[b_{11}, b_{22}, \ldots, b_{p p}\right]$, the matrix $G(x) B$ can be decomposed as follows:

$$
G(x) B=G_{b s}(x) D_{b} T_{b}(x),
$$

where $G_{b s}(x) \in R^{P \times P}$ is a symmetric positive-definite matrix, $D_{b} \in R^{P \times P}$ is a diagonal matrix whose elements are +1 or -1 and $\mathrm{T}_{b}(x) \in R^{P \times P}$ is a unity upper triangular matrix. Moreover, the diagonal elements $d_{b i i}$ of $D_{b}$ are nothing else than the ratios of the signs of the leading principal minors of $G(x)$ (i.e. $\left.D_{b}=D\right)$.

Proof of Lemma 2. In this proof, we will exploit directly the results of Lemma 1. It consists on two parts.

(1) Part 1:

We start by showing the existence of this composition. According to Lemma 1, the decomposition (24) exists, if the matrix $G(x) B$ has non-zero leading principal minors.

Since $B$ is a diagonal matrix, thus:

$$
G(x) B=\left[\begin{array}{lll}
b_{11} g_{11}(x) & \ldots & b_{p p} g_{1 p}(x) \\
\vdots & \ddots & \vdots \\
b_{11} g_{p 1}(x) & \ldots & b_{p p} g_{p p}(x)
\end{array}\right],
$$

The calculation of the minors of the matrix $G(x) B$ gives

$$
\begin{aligned}
\Delta_{b 1} & =b_{11} \Delta_{1}, \\
\Delta_{b 2} & =b_{11} b_{22} \Delta_{2}, \\
\vdots & \\
\Delta_{b p} & =b_{11} b_{22} \ldots b_{p p} \Delta_{p},
\end{aligned}
$$

where $\Delta_{i}$, with $i=1, \ldots, p$, are the minors of the matrix $G(x)$.

From (26) and since $B$ is a positive-definite matrix, thus its diagonal elements are strictly positive (i.e. $\left.b_{i i}>0, \forall i=1, \ldots, p\right)$, thus all leading principal minors of the matrix $G(x) B$ are non-zero and hence the decomposition (24) exists.

(2) Part 2:

Now, we show that $D_{b}=D$.

According to Lemma 1, diagonal elements of $D_{b}$ are nothing than the ratios of the signs of the leading principal minors of $G(x) B$, i.e.

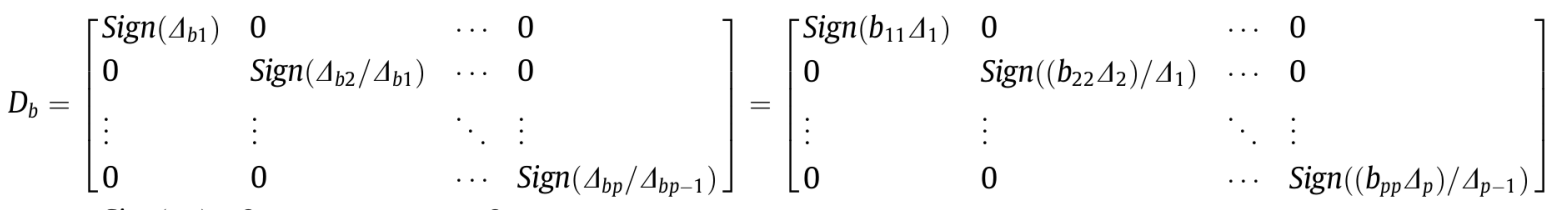

$$
\begin{aligned}
& =\left[\begin{array}{llll}
\operatorname{Sign}\left(\Delta_{1}\right) & 0 & \cdots & 0 \\
0 & \operatorname{Sign}\left(\Delta_{2} / \Delta_{1}\right) & \cdots & 0 \\
\vdots & \vdots & \ddots & \vdots \\
0 & 0 & \cdots & \operatorname{Sign}\left(\Delta_{p} / \Delta_{p-1}\right)
\end{array}\right]=D .
\end{aligned}
$$

This ends the proof of Lemma 2.

\subsection{Nussbaum function}

In order to cope with the unknown control direction, the Nussbaum gain technique will be used to construct the desired controllers in Sections 3.1 and 3.2. A function $N(\zeta)$ is called a Nussbaum function, if it has the following properties [17,37]:

(1) $\lim _{s \rightarrow+\infty} \sup \frac{1}{s} \int_{0}^{s} N(\zeta) d \zeta=+\infty$.

(2) $\lim _{s \rightarrow+\infty} \inf \frac{1}{s} \int_{0}^{s} N(\zeta) d \zeta=-\infty$. 
Example 1. The following functions are Nussbaum functions [17,37,62]:

$$
N_{1}(\zeta)=\zeta^{2} \cos (\zeta), \quad N_{2}(\zeta)=\zeta \cos (\sqrt{|\zeta|}), \quad \text { and } \quad N_{3}(\zeta)=\cos \left(\frac{\pi}{2} \zeta\right) e^{\zeta^{2}} .
$$

Of course, the cosine in the above examples can be replaced by the sine. It is easy to see that $N_{1}(\zeta), N_{2}(\zeta)$, and $N_{3}(\zeta)$ are Nussbaum functions. For clarity, the even Nussbaum function $N(\zeta)=\cos \left(\frac{\pi}{2} \zeta\right) e^{\zeta^{2}}$ will be used throughout this paper.

Thereafter, we need the following lemma in the stability analysis.

Lemma $3([17,63])$. Let $V(\cdot)$ and $\zeta(\cdot)$ be smooth functions defined on $\left[0, t_{f}\right)$, with $V(t) \geqslant 0, \forall t \in\left[0, t_{f}\right)$, and $N(\cdot)$ be an even Nussbaum function. If the following inequality holds:

$$
V(t) \leqslant c_{0}+\int_{0}^{t}(g N(\zeta)+1) \dot{\zeta} d \tau, \quad \forall t \in\left[0, t_{f}\right)
$$

where $g$ is a non-zero constant and $c_{0}$ represents some suitable constant, then $V(t), \zeta(t)$ and $\int_{0}^{t}(g N(\zeta)+1) \dot{\zeta} d \tau$ must be bounded on $\left[0, t_{f}\right)$.

Proof of Lemma 3. See the proof in [17].

\section{Design of fuzzy adaptive controllers}

Two fuzzy adaptive control schemes are developed in this section for MIMO uncertain nonlinear systems with both unknown actuator nonlinearities and unknown control direction. The design of the first control scheme is particularly carried out in the free time-delay case, while the second control scheme is performed assuming that the system exhibits time-varying delays.

\subsection{Design of a fuzzy adaptive controller for systems without time-delays in states}

In this section, we assume that $h_{\tau i}\left(x_{\tau}\right)=0$, i.e. the system (1) is supposed free of time-delays. Using the matrix decomposition (23), the system (2) can be rewritten as follows:

$$
y^{(r)}=F(x)+G_{s}(x) D T(x) u .
$$

To facilitate the control design and the stability analysis, the following realistic assumptions are considered

\section{Assumption 3}

(a) The sign of $G(x)$ is unknown and it must be positive-definite or negative-definite.

(b) $G_{s}(x)$ and $\frac{d}{d t} G_{s}^{-1}(x)$ are continuous functions.

(c) $\partial g_{i j}(x) / \partial y_{j}^{\left(r_{j}-1\right)}=0, \forall i=1,2, \ldots, p$, and $j=1,2, \ldots, p$.

\section{Remark 1}

(a) Assumption 3 is satisfied by many physical systems, namely robot-manipulators and induction machines.

(b) The required property on the partial derivatives of the control gain matrix ensures that the time derivative of $G_{s}^{-1}(x)$ depends only on the state vector $x$ (i.e. it ensures that $d G_{s}^{-1}(x) / d t$ does not depend on the system input).

\subsubsection{Fuzzy adaptive control design}

From Eqs. (28) and (20), the dynamic of $S$ can be rewritten as follows:

$$
G_{s}^{-1}(x) \dot{S}=G_{s}^{-1}(x)\left[C_{r}^{T} E+y_{d}^{(r)}-F(x)\right]-D T(x) u .
$$

Posing $G_{1}(x)=G_{s}^{-1}(x), F_{1}(x, u)=G_{s}^{-1}(x)\left[C_{r}^{T} E+y_{d}^{(r)}-F(x)\right]-[D T(x)-D] u$, Eq. (29) becomes

$$
G_{1}(x) \dot{S}=F_{1}(x, u)-D u .
$$

Using (22), (30) can be rearranged as follows :

$$
G_{2}(x) \dot{S}=-\frac{1}{2} \dot{G}_{2} S+\alpha(\bar{z})-D v-B^{-1} D d(v),
$$


where $G_{2}(x)=B^{-1} G_{1}(x)$ and $\alpha(\bar{z})=\left[\alpha_{1}\left(\bar{z}_{1}\right), \alpha_{2}\left(\bar{z}_{2}\right), \ldots, \alpha_{p}\left(\bar{z}_{P}\right)\right]^{T}=B^{-1} F_{1}(x, u)+\frac{1}{2} \dot{G}_{2}(x) S$, with $\bar{z}=\left[\bar{z}_{1}^{T}, \bar{z}_{2}^{T}, \ldots, \bar{z}_{p}^{T}\right]^{T}$, the vectors $\bar{z}_{i}$ will be defined in (32).

It is clear that since $B^{-1}$ is a diagonal positive-definite matrix and $G_{1}(x)$ is a symmetric positive-definite matrix, then the resultant matrix $G_{2}(x)=B^{-1} G_{1}(x)$ is also positive-definite but not necessary symmetric. In order to preserve this useful property (i.e. the symmetry property) which will be exploited later in the stability analysis, the following assumption is made on the matrix $B$ :

Assumption 4. All diagonal elements of $B$ are equal, i.e. $b_{11}=b_{22}=\cdots=b_{p p}$.

Note that, in the general case, this assumption can be considered relatively restrictive. It will be relaxed in Section 3.2. By carefully examining the expressions of $F_{1}(x, u), u=N(v)$ and $\alpha(\bar{z})$, the vectors $\bar{z}_{i}$ can be determined as follows:

$$
\begin{gathered}
\bar{z}_{1}=\left[x^{T}, S^{T}, v_{2}, \ldots, v_{p}\right]^{T}, \\
\bar{z}_{2}=\left[x^{T}, S^{T}, v_{3}, \ldots, v_{p}\right]^{T}, \\
\quad \vdots \\
\bar{z}_{p-1}=\left[x^{T}, S^{T}, v_{P}\right]^{T}, \\
\bar{z}_{p}=\left[x^{T}, S^{T}\right]^{T} .
\end{gathered}
$$

It is clear from the property of the matrix $D T(x)-D$ that $\bar{z}_{1}$ depends on control inputs $v_{2}, \ldots, v_{p}, \bar{z}_{2}$ depends on $v_{3}, \ldots, v_{p}$ and so on. The nonlinearity $\alpha(\bar{z})$ has an upper triangular control structure, allowing thereby for algebraic loop free sequential determination of the control variables $v_{i}$, for $i=1,2, \ldots, p$.

Let us define the following compact sets

$$
\begin{aligned}
& \Omega_{z_{i}}=\left\{\left[x^{T}, S^{T}, v_{i+1}, \ldots, v_{p}\right]^{T} \mid x \in \Omega_{x} \subset R^{r}, x_{d} \in \Omega_{x_{d}}\right\}, \quad \text { for } i=1,2, \ldots, p-1, \quad \text { and } \\
& \Omega_{z_{p}}=\left\{\left[x^{T}, S^{T}\right] \mid x \in \Omega_{x} \subset R^{r}, x_{d} \in \Omega_{x_{d}}\right\} .
\end{aligned}
$$

Remark 2. The choice of the vectors $\bar{z}_{i}$, i.e. the input arguments of the unknown functions $\alpha_{i}$, is not unique. Indeed, since $S$ and $v$ are functions of $x$ and $x_{d}$, then it can be easily seen that all vectors $\bar{z}_{i}$ are functions of $x$ and $x_{d}$, e.g. we can choose $\bar{z}_{i}=\left[x^{T}, x_{d}^{T}\right]^{T}$ or $\bar{z}_{i}=\left[x^{T}, E^{T}\right]^{T}, \bar{z}_{i}=\left[x^{T}, S^{T}\right]^{T}$ with $i=1, \ldots, p$.

The unknown nonlinear function $\alpha_{i}\left(\bar{z}_{i}\right)$ can be approximated, on the compact set $\Omega_{z_{i}}$, by the linearly parameterized fuzzy systems used in $[3,4]$, as follows:

$$
\hat{\alpha}_{i}\left(\bar{z}_{i}, \theta_{i}\right)=\theta_{i}^{T} \psi_{i}\left(\bar{z}_{i}\right), \quad i=1, \ldots, p,
$$

where $\psi_{i}\left(\bar{z}_{i}\right)$ is the fuzzy basis function (FBF) vector, which is fixed a priori by the designer, and $\theta_{i}$ is the adjustable parameter vector of the fuzzy system. For more details regarding the fuzzy system used here, see $[3,4]$.

Let us define

$$
\theta_{i}^{*}=\arg \min _{\theta_{i}}\left[\sup _{\bar{z}_{i} \in \Omega_{z_{i}}}\left|\alpha_{i}\left(\bar{z}_{i}\right)-\hat{\alpha}_{i}\left(\bar{z}_{i}, \theta_{i}\right)\right|\right]
$$

as the optimal values of $\theta_{i}$ which are mainly introduced for analysis purposes as their values are not needed when implementing the controller.

Define

$$
\tilde{\theta}_{i}=\theta_{i}-\theta_{i}^{*} \quad \text { and } \quad \varepsilon_{i}\left(\bar{z}_{i}\right)=\alpha_{i}\left(\bar{z}_{i}\right)-\hat{\alpha}_{i}\left(\bar{z}_{i}, \theta_{i}^{*}\right) \quad \text { with } \hat{\alpha}_{i}\left(\bar{z}_{i}, \theta_{i}^{*}\right)=\theta_{i}^{* T} \psi_{i}\left(\bar{z}_{i}\right)
$$

as the parameter estimation error and the fuzzy approximation error, respectively.

As in the literature $[3-6,9-12,33,53,54,56,60]$, we assume that the used fuzzy systems do not violate the universal approximator property on the compact set $\Omega_{z_{i}}$, which is assumed large enough so that the input vector of the fuzzy system remains in $\Omega_{z_{i}}$ under the closed-loop control system. It is hence reasonable to assume that the fuzzy approximation error is bounded for all $\bar{z}_{i} \in \Omega_{z_{i}}$, i.e.

$$
\left|\varepsilon_{i}\left(\bar{z}_{i}\right)\right| \leqslant \bar{\varepsilon}_{i}, \quad \forall \bar{z}_{i} \in \Omega_{z_{i}},
$$

where $\bar{\varepsilon}_{i}$ is an unknown constant. 
Now, let us denote

$$
\begin{aligned}
& \hat{\alpha}(\bar{z}, \theta)=\left[\hat{\alpha}_{1}\left(\bar{z}_{1}, \theta_{1}\right) \ldots \hat{\alpha}_{p}\left(\bar{z}_{p}, \theta_{p}\right)\right]^{T} \\
& \varepsilon(\bar{z})=\left[\varepsilon_{1}\left(\bar{z}_{1}\right) \ldots \varepsilon_{p}\left(\bar{z}_{p}\right)\right]^{T} \\
& \bar{\varepsilon}=\left[\bar{\varepsilon}_{1} \ldots \bar{\varepsilon}_{p}\right]^{T} .
\end{aligned}
$$

From the above analysis, we have

$$
\begin{aligned}
\hat{\alpha}(\bar{z}, \theta)-\alpha(\bar{z}) & =\hat{\alpha}(\bar{z}, \theta)-\hat{\alpha}\left(\bar{z}, \theta^{*}\right)+\hat{\alpha}\left(\bar{z}, \theta^{*}\right)-\alpha(\bar{z}), \\
& =\hat{\alpha}(\bar{z}, \theta)-\hat{\alpha}\left(\bar{z}, \theta^{*}\right)-\varepsilon(\bar{z}), \\
& =\tilde{\theta}^{T} \psi(\bar{z})-\varepsilon(\bar{z}) .
\end{aligned}
$$

where $\tilde{\theta}^{T} \psi(\bar{z})=\left[\tilde{\theta}_{1}^{T} \psi_{1}\left(\bar{z}_{1}\right), \tilde{\theta}_{2}^{T} \psi_{2}\left(\bar{z}_{2}\right), \ldots, \tilde{\theta}_{p}^{T} \psi_{p}\left(\bar{z}_{p}\right)\right]^{T}$, and $\tilde{\theta}_{i}=\theta_{i}-\theta_{i}^{*}, i=1, \ldots, p$.

Consider the following fuzzy adaptive controller which incorporates a Nussbaum function

$$
v=N(\zeta)\left[-\hat{\alpha}(\bar{z}, \theta)-K_{0} \operatorname{Sign}(S)-K_{1} S\right]=N(\zeta)\left[-\theta^{T} \psi(\bar{z})-K_{0} \operatorname{Sign}(S)-K_{1} S\right],
$$

and

$$
\dot{\zeta}=\sum_{i=1}^{p}\left[\theta_{i}^{T} \psi_{i}\left(\bar{z}_{i}\right)+k_{0 i} \operatorname{Sign}\left(S_{i}\right)+k_{1 i} S_{i}\right] S_{i},
$$

where $N(\zeta)=\cos \left(\frac{\pi}{2} \zeta\right) e^{\zeta^{2}}, K_{0}=\operatorname{Diag}\left[k_{01}, k_{02}, \ldots, k_{0 p}\right]$ and $K_{1}=\operatorname{Diag}\left[k_{11}, k_{12}, \ldots, k_{1 p}\right] . k_{1 i}>0$ for $i=1, \ldots, p$ are design constants and $k_{0 i}$ for $i=1, \ldots, p$ are the online estimates of the uncertain terms $\frac{1}{2} \sigma_{i}\left\|\theta_{i}^{*}\right\|^{2}+b_{i i}^{-1} d_{i}^{*}+\bar{\varepsilon}_{i}$. The parameter $\sigma_{i}$ will be defined below Eq. (41).

The corresponding adaptive laws are

$$
\begin{aligned}
& \dot{\theta}_{i}=-\sigma_{i} \gamma_{1 i}\left|S_{i}\right| \theta_{i}+\gamma_{1 i} S_{i} \psi_{i}\left(\bar{z}_{i}\right), \\
& \dot{k}_{0 i}=\gamma_{0 i}\left|S_{i}\right|,
\end{aligned}
$$

where $\gamma_{1 i}, \gamma_{0 i}$ and $\sigma_{i}>0$ are design constants. The term $\sigma_{i} \gamma_{1 i}\left|S_{i}\right| \theta_{i}$, borrowed from the $e$-modification concept [36], is mainly motivated by parameter boundedness purposes.

Note that the control law (38) is mainly composed of three control terms: a fuzzy adaptive term $\theta^{T} \psi(\bar{z})$ which is used to cancel the nonlinearities $\alpha(\bar{z})$ and a robust control term $K_{0} \operatorname{Sign}(S)$ which is introduced to compensate for the fuzzy approximation errors $\varepsilon(\bar{z})$ and to eliminate the effects of both the actuator nonlinearities $B^{-1} D d(v)$ and the terms $\frac{1}{2} \sigma_{i}\left\|\theta_{i}^{*}\right\|^{2}$ due to the use of the e-modification in the adaptation law (40). As for $K_{1} S$, it is used for the stability purposes. It is also worth mentioning that the Nussbaum gain function $N(\zeta)$ is used to estimate the true control direction.

Using the control law (38) and the expression (37), (31) becomes

$$
\begin{aligned}
G_{2}(x) \dot{S} & =-\frac{1}{2} \dot{G}_{2} S-K_{1} S-K_{0} \operatorname{Sign}(S)-\tilde{\theta}^{T} \psi(\bar{z})+\varepsilon(\bar{z})+\left[\theta^{T} \psi(\bar{z})+K_{0} \operatorname{Sign}(S)+K_{1} S\right]-D v-B^{-1} \operatorname{Dd}(v), \\
& =-\frac{1}{2} \dot{G}_{2} S-K_{1} S-K_{0} \operatorname{Sign}(S)-\tilde{\theta}^{T} \psi(\bar{z})+\left[\theta^{T} \psi(\bar{z})+K_{0} \operatorname{Sign}(S)+K_{1} S\right][1+g N(\zeta)]+\varepsilon(\bar{z})-B^{-1} \operatorname{Dd}(v) .
\end{aligned}
$$

where $g=d_{i i}, \forall i=1,2, \ldots, p$, where $d_{i i}$ are the diagonal elements of $D$.

Multiplying (42) by $S^{T}$ yields

$$
\begin{aligned}
S^{T} G_{2}(x) \dot{S}= & -\frac{1}{2} S^{T} \dot{G}_{2} S-S^{T} K_{1} S-\sum_{i=1}^{p} k_{0 i}\left|S_{i}\right|-S^{T} \tilde{\theta}^{T} \psi(\bar{z})+\sum_{i=1}^{p}\left(\varepsilon_{i}\left(\bar{z}_{i}\right)-b_{i i}^{-1} g d_{i}\left(v_{i}\right)\right) S_{i}+\sum_{i=1}^{P} S_{i}\left[\theta_{i}^{T} \psi_{i}\left(\bar{z}_{i}\right)+k_{0 i} \operatorname{Sign}\left(S_{i}\right)\right. \\
& \left.+k_{1 i} S_{i}\right]+g N(\zeta)\left(\sum_{i=1}^{P} S_{i}\left[\theta_{i}^{T} \psi_{i}\left(\bar{z}_{i}\right)+k_{0 i} \operatorname{Sign}\left(S_{i}\right)+k_{1 i} S_{i}\right]\right) \\
= & -\frac{1}{2} S^{T} \dot{G}_{2} S-S^{T} K_{1} S-\sum_{i=1}^{p} k_{0 i}\left|S_{i}\right|-S^{T} \tilde{\theta}^{T} \psi(\bar{z})+\sum_{i=1}^{p}\left(\varepsilon_{i}\left(\bar{z}_{i}\right)-b_{i i}^{-1} g d_{i}\left(v_{i}\right)\right) S_{i}+\dot{\zeta}+g N(\zeta) \dot{\zeta} .
\end{aligned}
$$

\subsubsection{Stability analysis}

Theorem 1. Consider the system (1) which is assumed to be free of the time-delays and with Assumptions 1-4. Then, the control law defined by Eqs. (38)-(41) guarantees the following properties:

- All variables involved in the control system are bounded.

- The tracking errors and their derivatives decrease asymptotically to zero, i.e. $e_{i}^{(j)}(t) \rightarrow 0$ as $t \rightarrow \infty$ for $i=1, \ldots, p$ and $j=0,1, \ldots, r_{i}-1$. 
Proof of Theorem 1. It is carried out using the following Lyapunov function candidate:

$$
V=\frac{1}{2} S^{T} G_{2}(x) S+\frac{1}{2} \sum_{i=1}^{p} \frac{1}{\gamma_{1 i}} \tilde{\theta}_{i}^{T} \tilde{\theta}_{i}+\frac{1}{2} \sum_{i=1}^{p} \frac{1}{\gamma_{0 i}}\left(\tilde{k}_{0 i}\right)^{2},
$$

with $\tilde{k}_{0 i}=k_{0 i}-k_{0 i}^{*}$, where $k_{0 i}^{*}=0.5 \sigma_{i}\left\|\theta_{i}^{*}\right\|^{2}+b_{i i}^{-1} d_{i}^{*}+\bar{\varepsilon}_{i}$ and $k_{0 i}$ is the estimate of the unknown parameter $k_{0 i}^{*}$.

Its time derivative is given by:

$$
\dot{V}=S^{T} G_{2}(x) \dot{S}+\frac{1}{2} S^{T} \dot{G}_{2}(x) S+\sum_{i=1}^{p} \frac{1}{\gamma_{1 i}} \tilde{\theta}_{i}^{T} \dot{\theta}_{i}+\sum_{i=1}^{p} \frac{1}{\gamma_{0 i}} \tilde{k}_{0 i} \dot{k}_{0 i}
$$

Using (43), (40) and (41) and the fact that $2 \tilde{\theta}_{i}^{T} \theta_{i} \geqslant\left\|\tilde{\theta}_{i}\right\|^{2}-\left\|\theta_{i}^{*}\right\|^{2}$ yields

$$
\begin{aligned}
\dot{V} & \leqslant-\sum_{i=1}^{p} k_{1 i} S_{i}^{2}-\sum_{i=1}^{p} k_{0 i}\left|S_{i}\right|+\sum_{i=1}^{p} \bar{\varepsilon}_{i}\left|S_{i}\right|+\sum_{i=1}^{p} b_{i i}^{-1} d_{i}^{*}\left|S_{i}\right|+\sum_{i=1}^{p} \tilde{k}_{0 i}\left|S_{i}\right|+\frac{1}{2} \sum_{i=1}^{p} \sigma_{i}\left|S_{i}\right|\left\|\theta_{i}^{*}\right\|^{2}+\dot{\zeta}+g N(\zeta) \dot{\zeta} \\
& =-\sum_{i=1}^{p} k_{1 i} S_{i}^{2}-\sum_{i=1}^{p} k_{0 i}\left|S_{i}\right|+\sum_{i=1}^{p} k_{0 i}^{*}\left|S_{i}\right|+\sum_{i=1}^{p} \tilde{k}_{0 i}\left|S_{i}\right|+\dot{\zeta}+g N(\zeta) \dot{\zeta}=-\sum_{i=1}^{p} k_{1 i} S_{i}^{2}+\dot{\zeta}+g N(\zeta) \dot{\zeta} .
\end{aligned}
$$

Recall that $k_{0 i}^{*}=\frac{1}{2} \sigma_{i}\left\|\theta_{i}^{*}\right\|^{2}+b_{i i}^{-1} d_{i}^{*}+\bar{\varepsilon}_{i}, \tilde{k}_{0 i}=k_{0 i}-k_{0 i}^{*}$ and $|g|=\left|d_{i i}\right|=1$.

Integrating (46) over $[0, t]$, we have

$$
V(t) \leqslant V(t)+\int_{0}^{t} \sum_{i=1}^{p} k_{1 i} S_{i}^{2} d \tau \leqslant V(0)+\int_{0}^{t}(\dot{\zeta}+g N(\zeta) \dot{\zeta}) d \tau .
$$

According to Lemma 3, [17,63], we have $V(t), \int_{0}^{t}(1+g N(\zeta)) \dot{\zeta} d \tau$ and $\zeta$ are bounded on $\left[0, t_{f}\right)$. According to the discussion in $[17,63]$, we know that the above conclusion is also true for $t_{f}=+\infty$, therefore $S_{i}, \tilde{\theta}_{i}, \tilde{k}_{0 i} \in L_{\infty}$. Then, from the boundedness of $S_{i}, \tilde{\theta}_{i}, \tilde{k}_{0 i}$ and $\zeta$, we can easily conclude about the boundedness of $\theta_{i}, k_{0 i}$ and $v$. From (46) and since $\int_{0}^{\infty}(1+g N(\zeta)) \dot{\zeta} d \tau$ and $V(t)$ are bounded, it is very easy to show that $\int_{0}^{\infty} \sum_{i=1}^{p} S_{i}^{2} d t$ exists, i.e. $S_{i} \in L_{2}$.

In order to show the boundedness of $\dot{S}_{i}$, we rearrange (42) as follows:

$$
\dot{S}=G_{2}^{-1}(x)\left[-\frac{1}{2} \dot{G}_{2} S-K_{1} S-K_{0} \operatorname{Sign}(S)-\tilde{\theta}^{T} \psi(\bar{z})+\varepsilon(\bar{z})+\left[\theta^{T} \psi(\bar{z})+K_{0} \operatorname{Sign}(S)+K_{1} S\right]-D v-B^{-1} D d(v)\right] .
$$

From (48) and since $S_{i}, \tilde{\theta}_{i} \theta_{i}, v, x, \varepsilon(\bar{z}), K_{0}, d(v) \in L_{\infty}, G_{2}^{-1}(x)$ is a positive-definite matrix (i.e. $\exists \sigma_{0}>0$, such as $\left.\left\|G_{2}^{-1}(x)\right\| \geqslant \sigma_{0}\right)$, and $G_{2}^{-1}(x)$ and $\dot{G}_{1}(x)$ are continuous functions, we can easily show that $\dot{S}_{i} \in L_{\infty}$.

Finally, since $S_{i} \in L_{2} \cap L_{\infty}$ and $\dot{S}_{i} \in L_{\infty}$, we can conclude according to Barbalat's Lemma [40] that $S_{i}(t) \rightarrow 0$ as $t \rightarrow \infty$. This means that the tracking errors and their derivatives converge asymptotically to zero, i.e. $e_{i}^{(j)}(t) \rightarrow 0$ as $t \rightarrow \infty$ for $i=1, \ldots, p$ and $j=0,1, \ldots, r_{i}-1$.

Remark 3. The sign function appearing in Eq. (38) would be replaced by any equivalent smooth function such as tanh and arctan to deal with the chattering effects. However, with these continuous functions, the tracking errors can only converge to a neighborhood of the origin.

Remark 4. If the matrix $D$ is known, we do not need to use the Nussbaum function in the control law (38)-(41). In this case, this control law becomes:

$$
\begin{aligned}
& v=D\left[\theta^{T} \psi(\bar{z})+K_{0} \operatorname{Sign}(S)+K_{1} S\right], \\
& \dot{\theta}_{i}=-\sigma_{i} \gamma_{1 i}\left|S_{i}\right| \theta_{i}+\gamma_{1 i} S_{i} \psi_{i}\left(\bar{z}_{i}\right), \\
& \dot{k}_{0 i}=\gamma_{2 i}\left|S_{i}\right| .
\end{aligned}
$$

Note that the proof of the stability associated to the controller (49)-(51) and the convergence of tracking errors towards zero is straightforward.

\subsection{Design of a fuzzy adaptive controller for systems with delays in states}

Consider now the system (1) with Assumptions 1 and 2. In this section, the functions with time-delays, i.e. $h_{\tau i}\left(x_{\tau}\right)$, $i=1, \ldots, p$, are assumed to be different from zero. Moreover, Assumption 4 made on the matrix $B$ is removed.

From (2) and (20), we have

$$
\dot{S}=C_{r}^{T} E+y_{d}^{(r)}-F(x)-G(x) u-H_{\tau}\left(x_{\tau}\right) .
$$


Using Lemma 2 and (22), (52) becomes

$$
\dot{S}=C_{r}^{T} E+y_{d}^{(r)}-F(x)-G_{b s}(x) D_{b} T_{b}(x)\left(v+B^{-1} d(v)\right)-H_{\tau}\left(x_{\tau}\right) .
$$

In the sequel, we need the following realistic assumption.

\section{Assumption 5.}

(a) The sign of $G(x)$ is unknown but it must be positive-definite or negative-definite.

(b) $G_{b s}(x)$ and $\frac{d}{d t} G_{b s}^{-1}(x)$ are continuous functions.

(c) $\partial g_{i j}(x) / \partial y_{j}^{\left(r_{j}-1\right)}=0, \forall i=1,2, \ldots, p$, and $j=1,2, \ldots, p$.

The dynamics of $S$ (i.e. Eq. (53)) can be rewritten as:

$$
G_{b 1}(x) \dot{S}=F_{b 1}(x, v)-D_{b} v-D_{b} T_{b}(x) B^{-1} d(v)-G_{b 1}(x) H_{\tau}\left(x_{\tau}\right),
$$

where $G_{b 1}(x)=G_{b s}^{-1}(x), F_{b 1}(x, v)=G_{b s}^{-1}(x)\left[C_{r}^{T} E+y_{d}^{(r)}-F(x)\right]-\left[D_{b} T_{b}(x)-D_{b}\right] v$.

Similarly to Section 3.1 , we can rearrange (54) as follows:

$$
G_{b 1}(x) \dot{S}=-\frac{1}{2} \dot{G}_{b 1} S+\alpha(\bar{z})-D_{b} v-G_{b 1}(x) H_{\tau}\left(x_{\tau}\right)-D_{b} T_{b}(x) B^{-1} d(v),
$$

with $\alpha(\bar{z})=\left[\alpha_{1}\left(\bar{z}_{1}\right), \alpha_{2}\left(\bar{z}_{2}\right), \ldots, \alpha_{p}\left(\bar{z}_{P}\right)\right]^{T}=F_{b 1}(x, v)+\frac{1}{2} \dot{G}_{b 1}(x) S$, where $\bar{z}_{i}$ were already defined in Section 3.1.

Multiplying (55) by $S^{T}$, we have

$$
S^{T} G_{b 1}(x) \dot{S}=-\frac{1}{2} S^{T} \dot{G}_{b 1} S+S^{T} \alpha(\bar{z})-S^{T} D_{b} v-S^{T} G_{b 1}(x) H_{\tau}\left(x_{\tau}\right)-S^{T} D_{b} T_{b}(x) B^{-1} d(v) .
$$

Posing $G_{b 1}(x) H_{\tau}\left(x_{\tau}\right)=\bar{H}_{\tau}\left(x, x_{\tau}\right)=\left[\bar{h}_{\tau 1}\left(x, x_{\tau}\right), \ldots, \bar{h}_{\tau p}\left(x, x_{\tau}\right)\right]^{T}$, (56) becomes:

$$
S^{T} G_{b 1}(x) \dot{S}=-\frac{1}{2} S^{T} \dot{G}_{b 1} S+S^{T} \alpha(\bar{z})-S^{T} D_{b} v-\sum_{i=1}^{p} S_{i} \bar{h}_{\tau i}\left(x, x_{\tau}\right)-S^{T} D_{b} T_{b}(x) B^{-1} d(v) .
$$

Now let us denote

$$
\begin{aligned}
& \bar{E}=\left[\bar{E}_{1}^{T}, \ldots, \bar{E}_{p}^{T}\right]^{T}, \quad \text { with } \bar{E}_{i}=\left[e_{i}, \dot{e}_{i}, \ldots, e_{i}^{\left(r_{i}-2\right)}\right]^{T}=\left[e_{i 1}, e_{i 2} \ldots, e_{i, r_{i}-1}\right]^{T} \quad \text { for } i=1, \ldots, p ; \\
& \bar{E}_{\tau}=\left[\bar{E}_{\tau 1}^{T}, \ldots, \bar{E}_{\tau p}^{T}\right]^{T}, \quad \text { with } \bar{E}_{\tau i}=\left[e_{i 1}\left(t-\tau_{i}(t)\right), e_{i 2}\left(t-\tau_{i}(t)\right), \ldots, e_{i, r_{i}-1}\left(t-\tau_{i}(t)\right)\right]^{T} \quad \text { for } i=1, \ldots, p ; \\
& x_{d \tau}=\left[x_{d \tau 1}^{T}, \ldots, x_{d \tau p}^{T}\right]^{T}, \quad x_{d \tau i}=\left[y_{d i}\left(t-\tau_{i}(t)\right), \dot{y}_{d i}\left(t-\tau_{i}(t)\right), \ldots, y_{d i}^{\left(r_{i}-1\right)}\left(t-\tau_{i}(t)\right)\right]^{T}, \quad \text { for } i=1, \ldots, p .
\end{aligned}
$$

Eq. (57) can be expressed as follows:

$$
S^{T} G_{b 1}(x) \dot{S}=-\frac{1}{2} S^{T} \dot{G}_{b 1} S+S^{T} \alpha(\bar{z})-S^{T} D_{b} v-\sum_{i=1}^{p} S_{i}\left(\bar{h}_{\tau i}\left(x, x_{\tau}\right)-\bar{h}_{\tau i}\left(x, x_{d \tau}\right)\right)-S^{T} \bar{H}_{\tau}\left(x, x_{d \tau}\right)-S^{T} D_{b} T_{b}(x) B^{-1} d(v) .
$$

The following realistic assumptions are made.

Assumption 6. The unknown nonlinear functions $\bar{h}_{\tau i}\left(x_{\tau}\right)$ satisfy:

$$
\left|\bar{h}_{\tau i}\left(x, x_{\tau}\right)-\bar{h}_{\tau i}\left(x, x_{d \tau}\right)\right| \leqslant \bar{k}_{i}|| \bar{E}_{\tau} \|,
$$

where $\bar{k}_{i}$ is an unknown positive constant.

Assumption 7. We assume that

$$
\left|S^{T} \bar{H}_{\tau}\left(x, x_{d \tau}\right)+S^{T} D_{b} T_{b}(x) B^{-1} d(v)\right|=\left|S^{T} G_{b 1}(x) H_{\tau}\left(x_{d \tau}\right)+S^{T} D_{b} T_{b}(x) B^{-1} d(v)\right| \leqslant \sum_{i=1}^{p}\left|S_{i}\right| \rho_{i}(x)=|S|^{T} \rho(x),
$$

where $\rho_{i}(x)$ are unknown positive functions, $\rho(x)=\left[\rho_{1}(x), \ldots, \rho_{p}(x)\right]^{T}$ and $|S|^{T}=\left[\left|S_{1}\right|, \ldots,\left|S_{p}\right|\right]$.

Assumption 8. The time-varying delays $\tau_{i}(t)$ satisfy the following relations:

$$
0 \leqslant \tau_{i}(t) \leqslant \tau_{i}^{*}, \quad \dot{\tau}_{i}(t) \leqslant \bar{\tau}_{i}<1, \quad i=1, \ldots, p,
$$

where $\tau_{i}^{*}$ and $\bar{\tau}_{i}$ are unknown constants. 


\section{Remark 5}

(a) It is worth mentioning that the notations used below the Eq. (57) are only valid for $r_{i} \geqslant 2$. In the case where $r_{i}=1$, the vectors $\bar{E}_{i}$ and $\bar{E}_{\tau i}$ become as follows: $\bar{E}_{i}=e_{i 1}=e_{i}$ and $\bar{E}_{\tau i}=e_{i 1}\left(t-\tau_{i}(t)\right)$.

(b) Assumption 6 is generally adopted in the literature. It can be seen like a Lipschitz condition.

(c) Note that the continuous function $H_{\tau}\left(x_{d \tau}\right)$ is bounded since the signal $x_{d \tau}$ is bounded. Hence, the fact that $H_{\tau}\left(x_{d \tau}\right)$ and $d(v)$ are bounded, Assumption 7 is by no means restrictive and the functions $\rho_{i}(x)$ always exist.

(d) Assumption 8 is also an usual assumption in the control literature, see e.g. [24,62].

From (6), the dynamics of $\bar{E}_{i}$ can be expressed as follows:

$$
\dot{\bar{E}}_{i}=A_{c i} \bar{E}_{i}+B_{i} S_{i}, \quad \forall i=1, \ldots, 2
$$

where

$$
\begin{aligned}
A_{c i} & =\left[\begin{array}{lllll}
0 & 1 & 0 & \cdots & 0 \\
0 & 0 & 1 & \cdots & 0 \\
\vdots & \vdots & \vdots & \ddots & \vdots \\
0 & 0 & 0 & \cdots & 1 \\
-\lambda_{i}^{r_{i}-1} & -\left(r_{i}-1\right) \lambda_{i}^{r_{i}-2} & -\frac{1}{2}\left(r_{i}-1\right)\left(r_{i}-2\right) \lambda_{i}^{r_{i}-3} & \cdots & -\left(r_{i}-1\right) \lambda_{i}
\end{array}\right] \in R^{\left(r_{i}-1\right) \times\left(r_{i}-1\right)}, \\
B_{i} & =\left[\begin{array}{l}
0 \\
\vdots \\
0 \\
1
\end{array}\right] \in R^{\left(r_{i}-1\right)} .
\end{aligned}
$$

Since the matrix $A_{c i}$ is stable (i.e. Hurwitz), then for any given matrix $Q_{i}=Q_{i}^{T}>0$, there exists a single matrix $P_{i}=P_{i}^{T}>0$ being the solution of the following Lyapunov equation

$$
P_{i} A_{c i}+A_{c i}^{T} P_{i}=-Q_{i}
$$

The following fuzzy adaptive controller is proposed to achieve the control objective.

$$
\begin{aligned}
& v=N(\zeta)\left[-\theta^{T} \psi(\bar{z})-\left(K_{0}+W^{T} \varphi(x)\right) \operatorname{Sign}(S)-\left(K_{1}+K_{2}\right) S\right] \\
& \dot{\zeta}=\sum_{i=1}^{p}\left[\theta_{i}^{T} \psi_{i}\left(\bar{z}_{i}\right)+\left(k_{0 i}+W_{i}^{T} \varphi_{i}(x)\right) \operatorname{Sign}\left(S_{i}\right)+\left(k_{1 i}+k_{2 i}\right) S_{i}\right] S_{i}, \\
& \dot{\theta}_{i}=-\sigma_{i} \gamma_{1 i}\left|S_{i}\right| \theta_{i}+\gamma_{1 i} S_{i} \psi_{i}\left(\bar{z}_{i}\right), \\
& \dot{W}_{i}=-\delta_{i} \gamma_{3 i}\left|S_{i}\right| W_{i}+\gamma_{3 i}\left|S_{i}\right| \varphi_{i}(x) \\
& \dot{k}_{0 i}=\gamma_{0 i}\left|S_{i}\right| \\
& \dot{k}_{2 i}=\gamma_{2 i} S_{i}^{2}
\end{aligned}
$$

where $\gamma_{0 i}, \gamma_{1 i}, \gamma_{2 i}, \gamma_{3 i}, \sigma_{i}$, and $\delta_{i}$ are design positive constants, $K_{0}=\operatorname{Diag}\left[k_{01}, k_{02}, \ldots, k_{0 p}\right], K_{1}=\operatorname{Diag}\left[k_{11}, k_{12}, \ldots, k_{1 p}\right]$, and $K_{2}=\operatorname{Di-}$ $\operatorname{ag}\left[k_{21}, k_{22}, \ldots, k_{2 p}\right] . k_{1 i}>0$ for $i=1, \ldots, p$ are design constants, and $k_{2 i}$ and $k_{0 i}$ are the online estimates of the uncertain terms $k_{2 i}^{*}=\frac{\bar{k}_{i}^{2}}{2 l}+\left\|P_{i} B_{i}\right\|^{2}$ and $k_{0 i}^{*}=\frac{1}{2} \sigma_{i}\left\|\theta_{i}^{*}\right\|^{2}+\frac{1}{2} \delta_{i}\left\|W_{i}^{*}\right\|^{2}+\bar{\varepsilon}_{i}$, respectively. $l$ is an unknown positive constant which will be defined below (69). $\theta_{i}^{T} \psi_{i}\left(\bar{z}_{i}\right)$ and $W_{i}^{T} \varphi_{i}(x)$ are the estimates of the unknown nonlinearities $\alpha_{i}\left(\bar{z}_{i}\right)$ and $\rho_{i}(x)$, respectively. The term $\bar{\varepsilon}_{i}$ is the bound of fuzzy approximation errors and satisfies the following relation

$$
\bar{\varepsilon}_{i} \geqslant\left|\alpha_{i}\left(\bar{z}_{i}\right)-\theta_{i}^{* T} \psi_{i}\left(\bar{z}_{i}\right)\right|+\left|\rho_{i}(x)-W_{i}^{* T} \varphi_{i}(x)\right|,
$$

where $\theta_{i}^{*}$ and $W_{i}^{*}$ denote the optimal values of $\theta_{i}$ and $W_{i}$, respectively. 
Substituting the control law (63) into (58) leads to the following results

$$
\begin{aligned}
S^{T} G_{b 1}(x) \dot{S}+\frac{1}{2} S^{T} \dot{G}_{b 1} S \leqslant & -\sum_{i=1}^{p}\left[\theta_{i}^{T} \psi_{i}\left(\bar{z}_{i}\right)+\left(k_{0 i}+W_{i}^{T} \varphi_{i}(x)\right) \operatorname{Sign}\left(S_{i}\right)+\left(k_{1 i}+k_{2 i}\right) S_{i}\right] S_{i} \\
& +\sum_{i=1}^{p}\left[\alpha_{i}\left(\bar{z}_{i}\right) S_{i}+\rho_{i}(x)\left|S_{i}\right|\right]+\dot{\zeta}+g N(\zeta) \dot{\zeta}+\sum_{i=1}^{p} \bar{k}_{i}\left|S_{i}\right|\left\|\bar{E}_{\tau}\right\| \\
\leqslant & -\sum_{i=1}^{p} k_{1 i} S_{i}^{2}+\dot{\zeta}+g N(\zeta) \dot{\zeta}-\sum_{i=1}^{p} \tilde{\theta}_{i}^{T} \psi_{i}\left(\bar{z}_{i}\right) S_{i}-\sum_{i=1}^{p} \widetilde{W}_{i}^{T} \varphi_{i}\left(\bar{z}_{i}\right)\left|S_{i}\right| \\
& +\sum_{i=1}^{p} \bar{\varepsilon}_{i}\left|S_{i}\right|-\sum_{i=1}^{p} \tilde{k}_{0 i}\left|S_{i}\right|-\sum_{i=1}^{p} \tilde{k}_{2 i} S_{i}^{2}-\sum_{i=1}^{p} k_{0 i}^{*}\left|S_{i}\right|-\sum_{i=1}^{p} k_{2 i}^{*} S_{i}^{2}+\frac{1}{2} \sum_{i=1}^{p}\left(\bar{k}_{i}^{2} / l\right) S_{i}^{2}+\frac{1}{2} l p\left\|\bar{E}_{\tau}\right\|^{2},
\end{aligned}
$$

where $l=\min _{i}\left\{2\left(1-\bar{\tau}_{i}\right)\right\}, g=d_{b i i}, \forall i=1,2, \ldots, p$ (with $d_{b i i}$ are the diagonal terms of $D_{b}$ ), $\tilde{\theta}_{i}=\theta_{i}-\theta_{i}^{*}, \widetilde{W}_{i}=W_{i}-W_{i}^{*}$, $\tilde{k}_{0 i}=k_{0 i}-k_{0 i}^{*}$, and $\tilde{k}_{2 i}=k_{2 i}-k_{2 i}^{*}$.

Theorem 2. Consider the system (1) with Assumptions 1, 2 and Assumptions 5-8. Then, the control law defined by Eqs. (63)(68) guarantees the following properties:

- All signals in the closed-loop system are bounded.

- The tracking errors and their derivatives decrease asymptotically to zero, i.e. $e_{i}^{(j)}(t) \rightarrow 0$ as $t \rightarrow \infty$ for $i=1, \ldots, p$ and $j=0,1, \ldots, r_{i}-1$.

Proof of Theorem 2. Let us consider the following Lyapunov function candidate:

$$
V=V_{1}+V_{2}
$$

where

$$
\begin{aligned}
& V_{1}=\frac{1}{2} S^{T} G_{b 1}(x) S+\frac{1}{2} \sum_{i=1}^{p} \frac{1}{\gamma_{1 i}} \tilde{\theta}_{i}^{T} \tilde{\theta}_{i}+\frac{1}{2} \sum_{i=1}^{p} \frac{1}{\gamma_{3 i}} \widetilde{W}_{i}^{T} \widetilde{W}_{i}+\frac{1}{2} \sum_{i=1}^{p} \frac{1}{\gamma_{0 i}}\left(\tilde{k}_{0 i}\right)^{2}+\frac{1}{2} \sum_{i=1}^{p} \frac{1}{\gamma_{2 i}}\left(\tilde{k}_{2 i}\right)^{2}, \\
& V_{2}=\sum_{i=1}^{p} \bar{E}_{i}^{T} P_{i} \bar{E}_{i}+\sum_{i=1}^{p}\left(\frac{l p}{2\left(1-\bar{\tau}_{i}\right)} \int_{t-\tau_{i}(t)}^{t}\left\|\bar{E}_{i}(s)\right\|^{2} d s\right) .
\end{aligned}
$$

The time derivative of $V_{1}$ is given by:

$$
\dot{V}_{1}=S^{T} G_{b 1}(x) \dot{S}+\frac{1}{2} S^{T} \dot{G}_{b 1}(x) S+\sum_{i=1}^{p} \frac{1}{\gamma_{1 i}} \tilde{\theta}_{i}^{T} \dot{\theta}_{i}+\sum_{i=1}^{p} \frac{1}{\gamma_{3 i}} \widetilde{W}_{i}^{T} \dot{W}_{i}+\sum_{i=1}^{p} \frac{1}{\gamma_{0 i}} \tilde{k}_{0 i} \dot{k}_{0 i}+\sum_{i=1}^{p} \frac{1}{\gamma_{2 i}} \tilde{k}_{2 i} \dot{k}_{2 i}
$$

Using (69) and (64)-(68) and the fact that $2 \tilde{\theta}_{i}^{T} \theta_{i} \geqslant\left\|\tilde{\theta}_{i}\right\|^{2}-\left\|\theta_{i}^{*}\right\|^{2}$ and $2 \widetilde{W}_{i}^{T} W_{i} \geqslant\left\|\widetilde{W}_{i}\right\|^{2}-\left\|W_{i}^{*}\right\|^{2}$, (73) can be written as follows:

$$
\dot{V}_{1} \leqslant-\sum_{i=1}^{p} k_{1 i} S_{i}^{2}-\sum_{i=1}^{p} k_{2 i}^{*} S_{i}^{2}+\frac{1}{2} \sum_{i=1}^{p}\left(\bar{k}_{i}^{2} / l\right) S_{i}^{2}+\frac{1}{2} l p\left\|\bar{E}_{\tau}\right\|^{2}+\dot{\zeta}+g N(\zeta) \dot{\zeta}
$$

Recall that $k_{0 i}^{*}=\frac{1}{2} \sigma_{i}\left\|\theta_{i}^{*}\right\|^{2}+\frac{1}{2} \delta_{i}\left\|W_{i}^{*}\right\|^{2}+\bar{\varepsilon}_{i}$.

The time derivative of $V_{2}$ is

$$
\begin{aligned}
\dot{V}_{2} & =-\sum_{i=1}^{p} \bar{E}_{i}^{T} Q_{i} \bar{E}_{i}+\sum_{i=1}^{p} 2 \bar{E}_{i}^{T} P_{i} B_{i} S_{i}+\sum_{i=1}^{p} \frac{l p}{2\left(1-\bar{\tau}_{i}\right)}\left\|\bar{E}_{i}(t)\right\|^{2}-l p \sum_{i=1}^{p} \frac{1-\dot{\tau}_{i}(t)}{2\left(1-\bar{\tau}_{i}\right)}\left\|\bar{E}_{i}\left(t-\tau_{i}(t)\right)\right\|^{2} \\
& \leqslant-\sum_{i=1}^{p}\left(\lambda_{\min }\left(Q_{i}\right)-1\right)\left\|\bar{E}_{i}\right\|^{2}+\sum_{i=1}^{p}\left\|P_{i} B_{i}\right\|^{2} S_{i}^{2}+p \sum_{i=1}^{p}\left\|\bar{E}_{i}\right\|^{2}-l p \sum_{i=1}^{p} \frac{1-\dot{\tau}_{i}(t)}{2\left(1-\bar{\tau}_{i}\right)}\left\|\bar{E}_{i}\left(t-\tau_{i}(t)\right)\right\|^{2} \\
& =-\sum_{i=1}^{p}\left(\lambda_{\min }\left(Q_{i}\right)-(p+1)\right)\left\|\bar{E}_{i}\right\|^{2}+\sum_{i=1}^{p}\left\|P_{i} B_{i}\right\|^{2} S_{i}^{2}-\frac{1}{2} l p\left\|\bar{E}_{\tau}\right\|^{2} .
\end{aligned}
$$


Accordingly, we obtain

$$
\begin{aligned}
\dot{V} & =\dot{V}_{1}+\dot{V}_{2} \leqslant-\sum_{i=1}^{p} k_{1 i} S_{i}^{2}-\sum_{i=1}^{p} k_{2 i}^{*} S_{i}^{2}+\frac{1}{2} \sum_{i=1}^{p}\left(\bar{k}_{i}^{2} / l\right) S_{i}^{2}+\sum_{i=1}^{p}\left\|P_{i} B_{i}\right\|^{2} S_{i}^{2}+\dot{\zeta}+g N(\zeta) \dot{\zeta}-\sum_{i=1}^{p}\left(\lambda_{\min }\left(Q_{i}\right)-(p+1)\right)\left\|\bar{E}_{i}\right\|^{2} \\
& \leqslant-\sum_{i=1}^{p} k_{1 i} S_{i}^{2}+\dot{\zeta}+g N(\zeta) \dot{\zeta},
\end{aligned}
$$

where $k_{2 i}^{*}=\frac{k_{i}^{2}}{2 l}+\left\|P_{i} B_{i}\right\|^{2}$, and $\lambda_{\text {min }}\left(Q_{i}\right)$ is supposed to be selected large such as the term $\lambda_{\min }\left(Q_{i}\right)-(p+1)$ is always strictly positive. Note that the remainder of this proof is omitted since it is very similar to that of Theorem 1

\section{Simulation results}

Simulation studies are carried out to show the effectiveness of the proposed adaptive fuzzy controllers. Two control problems are considered to this end. The first one concerns a two-link rigid robot manipulator, while the second one concerns an academic MIMO nonlinear system with time-varying delays in states.

\subsection{Example 1}

In the following, we present simulation results showing the tracking performances of the first controller applied to a twolink rigid robot manipulator which moves in a horizontal plane. The dynamic equations of this MIMO system are given by Boulkroune et al. [5,6], and Slotine and Li [40]:

$$
\begin{aligned}
& \left(\begin{array}{l}
\ddot{q}_{1} \\
\ddot{q}_{2}
\end{array}\right)=\left(\begin{array}{ll}
M_{11} & M_{12} \\
M_{21} & M_{22}
\end{array}\right)^{-1}\left\{\left(\begin{array}{l}
u_{1} \\
u_{2}
\end{array}\right)-\left(\begin{array}{cc}
-h \dot{q}_{2} & -h\left(\dot{q}_{1}+\dot{q}_{2}\right) \\
h \dot{q}_{1} & 0
\end{array}\right)\left(\begin{array}{l}
\dot{q}_{1} \\
\dot{q}_{2}
\end{array}\right)\right\}, \\
& \left(\begin{array}{l}
u_{1} \\
u_{2}
\end{array}\right)=\left(\begin{array}{l}
N_{1}\left(v_{1}\right) \\
N_{2}\left(v_{2}\right)
\end{array}\right),
\end{aligned}
$$

where

$$
\begin{aligned}
& M_{11}=a_{1}+2 a_{3} \cos \left(q_{2}\right)+2 a_{4} \sin \left(q_{2}\right), \quad M_{22}=a_{2}, \\
& M_{21}=M_{12}=a_{2}+a_{3} \cos \left(q_{2}\right)+a_{4} \sin \left(q_{2}\right), \quad h=a_{3} \sin \left(q_{2}\right)-a_{4} \cos \left(q_{2}\right),
\end{aligned}
$$

with

$$
\begin{aligned}
& a_{1}=I_{1}+m_{1} l_{c 1}^{2}+I_{e}+m_{e} l_{c e}^{2}+m_{e} l_{1}^{2}, \quad a_{2}=I_{e}+m_{e} l_{c e}^{2}, \\
& a_{3}=m_{e} l_{1} l_{c e} \cos \left(\delta_{e}\right), \quad a_{4}=m_{e} l_{1} l_{c e} \sin \left(\delta_{e}\right),
\end{aligned}
$$

Note that $N_{i}\left(v_{i}\right)$ for $i=1,2$ are the considered input nonlinearities which can be dead-zones $D Z_{i}\left(v_{i}\right)$ or backlash hysteresis $B H_{i}\left(v_{i}\right)$. And $v_{i}$ for $i=1,2$ are the inputs of actuator nonlinearities, and $u_{i}$ for $i=1,2$ are their outputs.

The robot parameters are selected as follows:

$$
m_{1}=1, \quad m_{e}=2, \quad l_{1}=1, \quad l_{c 1}=0.5, \quad l_{c e}=0.6, \quad I_{1}=0.12, \quad I_{e}=0.25, \quad \delta_{e}=30^{\circ} .
$$

Let $\left[y_{1}, y_{2}\right]=\left[q_{1}, q_{2}\right], u=\left[u_{1}, u_{2}\right]^{T}=\left[N_{1}\left(v_{1}\right), N_{2}\left(v_{2}\right)\right]^{T}, x=\left[q_{1}, \dot{q}_{1}, q_{2}, \dot{q}_{2}\right]^{T}$. We can rewrite the robot model with actuator nonlinearities (Eq. (77)) as follows:

$$
\ddot{y}=F(x)+G(x) u,
$$

where

$$
\begin{aligned}
& F(x)=\left(\begin{array}{l}
f_{1}(x) \\
f_{2}(x)
\end{array}\right)=-M^{-1}\left(\begin{array}{ll}
-h \dot{q}_{2} & -h\left(\dot{q}_{1}+\dot{q}_{2}\right) \\
h \dot{q}_{1} & 0
\end{array}\right)\left(\begin{array}{l}
\dot{q}_{1} \\
\dot{q}_{2}
\end{array}\right), \\
& G(x)=\left(\begin{array}{ll}
g_{11}(x) & g_{12}(x) \\
g_{21}(x) & g_{22}(x)
\end{array}\right)=M^{-1}=\left(\begin{array}{ll}
M_{11} & M_{12} \\
M_{21} & M_{22}
\end{array}\right)^{-1} .
\end{aligned}
$$

The control objective consists in allowing the system outputs $q_{1}$ and $q_{2}$ to respectively track the sinusoidal desired trajectories $y_{d 1}=\sin (t)$ and $y_{d 2}=\sin (t)$.

Recall that the system nonlinearities $F(x)$ and $G(x)$, the sign of the matrix $G(x)$ and the parameters of the actuator nonlinearities are assumed to be completely unknown. In fact, the dynamic model (77) is only required for simulation purposes. 
The fuzzy system $\theta_{2}^{T} \psi_{2}\left(\bar{z}_{2}\right)$ has the vector $\left[q_{1}, \dot{q}_{1}, q_{2}, \dot{q}_{2}\right]^{T}$ as input, while $\theta_{1}^{T} \psi_{1}\left(\bar{z}_{1}\right)$ has the vector $\left[q_{1}, \dot{q}_{1}, q_{2}, \dot{q}_{2}, v_{2}\right]^{T}$ as input. For each variable of the entries of the fuzzy systems, as in [4], we define three (one triangular and two trapezoidal) membership functions uniformly distributed on the intervals $[-2,2]$ for $q_{1}, \dot{q}_{1}, q_{2}$, and $\dot{q}_{2}$, and $[-25,25]$ for $v_{2}$. The design parameters are chosen as follows: $\gamma_{11}=\gamma_{12}=400, \gamma_{01}=\gamma_{02}=5, \sigma_{1}=\sigma_{2}=0,05, \lambda_{1}=\lambda_{2}=2, k_{11}=k_{12}=2$. The initial conditions are selected as: $x(\mathbf{0})=\left[\begin{array}{llll}0.5 & 0 & 0.5 & 0\end{array}\right], k_{01}(\mathbf{0})=k_{02}(0)=0, \theta_{1}(0)=\underline{0}$ and $\theta_{2}(0)=\underline{0}$, where $\underline{0}$ is a null vector.

(a) Simulation with dead-zone nonlinearity in the input

The dead-zone model used here is described by [6,58]:

$$
u_{i}=N_{i}\left(v_{i}\right)= \begin{cases}m_{r i}\left(v_{i}-b_{r i}\right), & \text { for } v_{i} \geqslant b_{r i}, \\ 0, & \text { for } b_{l i}<v_{i}<b_{r i}, \\ m_{l i}\left(v_{i}-b_{l i}\right), & \text { for } v_{i} \leqslant b_{l i},\end{cases}
$$

where $b_{r i}>0, b_{l i}<0$ and $m_{r i}>0, m_{l i}>0$ are parameters and slopes of the dead-zone, respectively. As in [6,58], we can easily show that this model can be rewritten as (21).

The parameters of this dead-zone model are selected as follows: $b_{r 1}=b_{r 2}=3, b_{l 1}=b_{l 2}=-2.25, m_{1}=m_{2}=2$.

Fig. 1 shows simulation results of the first fuzzy adaptive controller. In spite of the presence of the dead-zone, these results show good tracking performances with the boundedness of the closed-loop system signals. Fig. 1(a) and (b) show that the tracking errors are bounded and converge to zero. Fig. 1(c) illustrates the boundedness of the control signals $\left(v_{i}\right)$. Fig. 1(d) also shows the control signals $\left(v_{i}\right)$ but for $t \in[0.5 \mathrm{~s}, 10 \mathrm{~s}]$.

(b) Simulation with backlash hysteresis in the input

Consider the backlash-like hysteresis used in $[5,42,43,59,65]$ and described by a continuous-time dynamic model

$$
\frac{d u_{i}}{d t}=a_{i}\left|\frac{d v_{i}}{d t}\right|\left(c_{i} v_{i}-u_{i}\right)+h_{i} \frac{d v_{i}}{d t},
$$

where $a_{i}, c_{i}$, and $h_{i}$ are constants, $c_{i}>0$ is the slope of the lines satisfying $c_{i}>h_{i} . u_{i}, v_{i}$ are the output and input of backlash, respectively. As $[5,42,43,59,65]$, we can easily show that this model can be rewritten as (21).

The parameters of this backlash are selected as follows: $a_{1}=a_{2}=0.5, c_{1}=c_{2}=3.1635$, and $h_{1}=h_{2}=0.345$.

Fig. 2 shows simulation results of the first fuzzy adaptive controller by taking into account the backlash hysteresis. From these simulation results, we can verify that this fuzzy adaptive controller is effective to cope with the hysteresis nonlinearity.

\subsection{Example 2}

In this section, we present simulation results showing the performances of the second controller applied to an academic MIMO nonlinear time-varying delays system. The dynamics of this MIMO system are given by:
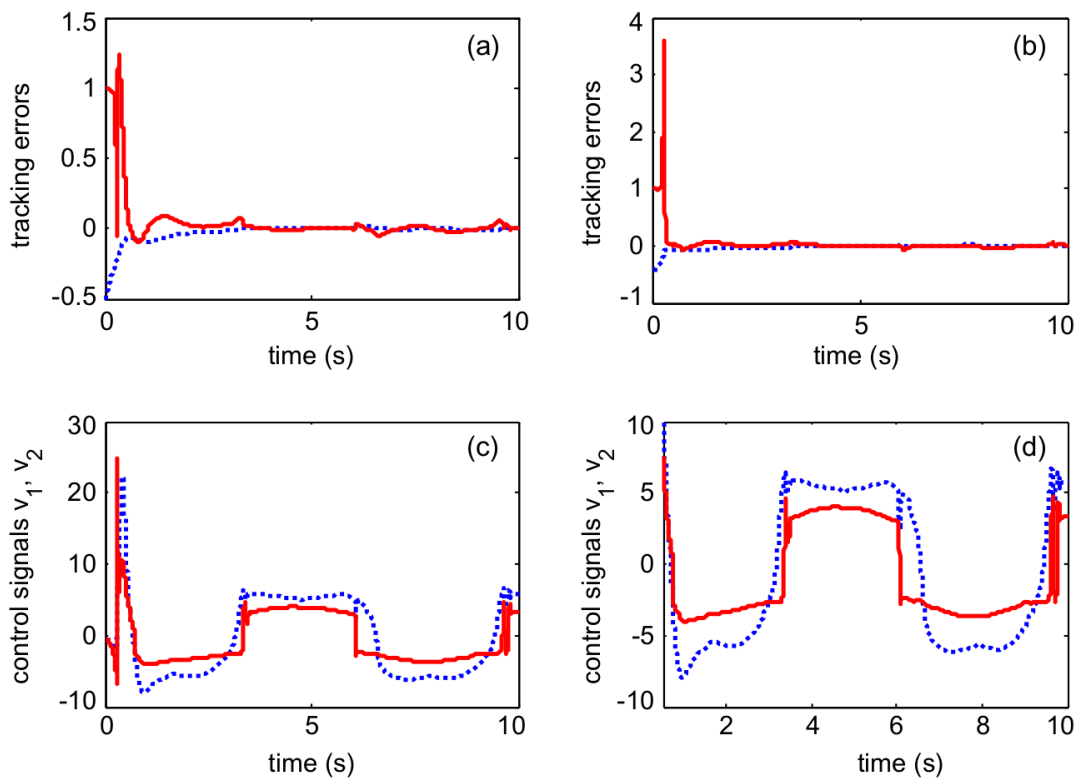

Fig. 1. Simulation results with dead-zone. (a) Tracking errors of link $1: e_{1}$ (dotted line) and $\dot{e}_{1}$ (solid line). (b) Tracking errors of link $2: e_{2}$ (dotted line) and $\dot{e}_{2}$ (solid line). (c) Control input signals: $v_{1}$ (dotted line) and $v_{2}$ (solid line). (d) Control input signals for $t \in[0.5,10]: v_{1}$ (dotted line) and $v_{2}$ (solid line). 

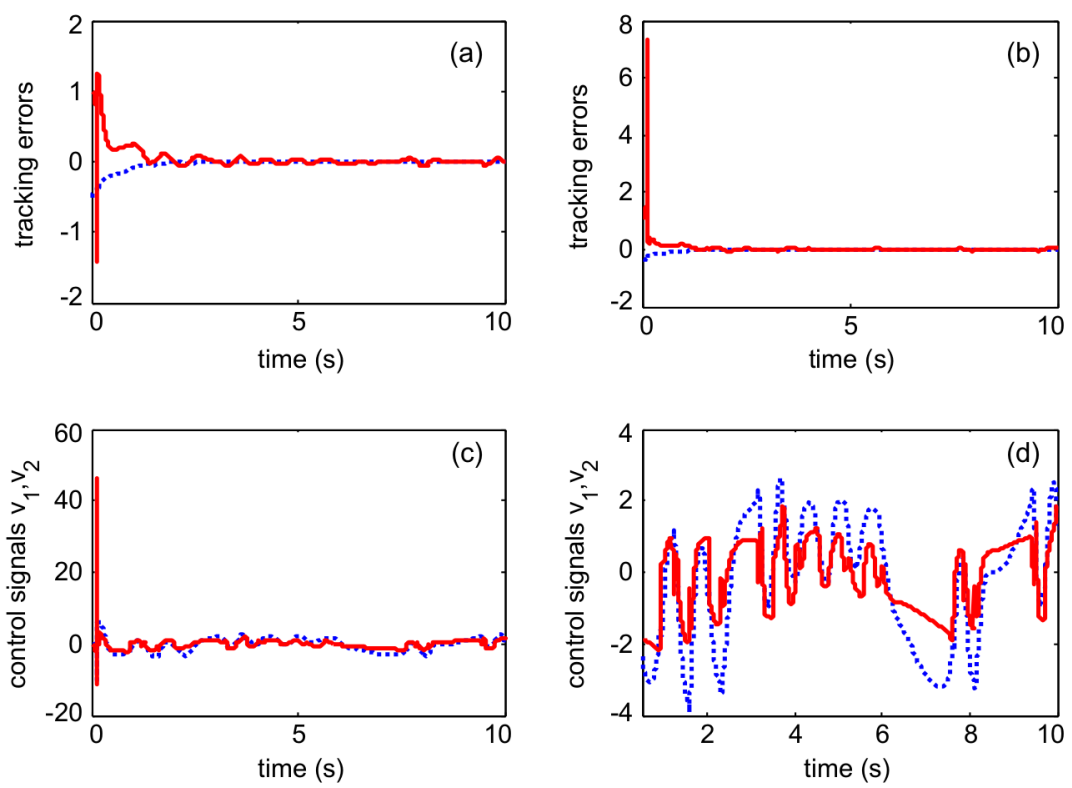

Fig. 2. Simulation results with backlash. (a) Tracking errors of link $1: e_{1}$ (dotted line) and $\dot{e}_{1}$ (solid line). (b) Tracking errors of link $2: e_{2}$ (dotted line) and $\dot{e}_{2}$ (solid line). (c) Control input signals: $v_{1}$ (dotted line) and $v_{2}$ (solid line). (d) Control input signals for $t \in[0.5,10]: v_{1}$ (dotted line) and $v_{2}$ (solid line).

$$
\left\{\begin{array}{l}
\dot{x}_{11}=x_{12} \\
\dot{x}_{12}=x_{21}-0.3 \sin \left(x_{11} x_{12}\right)+x_{12}^{2}+\left(2+\cos \left(x_{11}\right)\right) u_{1}+\left(1+x_{21}^{2}\right) u_{2}+0.2 x_{11}\left(t-\tau_{1}(t)\right) \sin \left(x_{11}\left(t-\tau_{1}(t)\right)\right) \\
\dot{x}_{21}=x_{22} \\
\dot{x}_{22}=x_{22}^{2}+x_{11}+x_{12}^{2}-u_{1}+\left(1+x_{21}^{2}\right) u_{2}+0.3 x_{21}\left(t-\tau_{2}(t)\right) \\
y_{1}(t)=x_{11}(t), \quad y_{2}(t)=x_{21}(t)
\end{array}\right.
$$

Let $y=\left[y_{1}, y_{2}\right]^{T}, u=\left[u_{1}, u_{2}\right]^{T}=\left[N_{1}\left(v_{1}\right), N_{2}\left(v_{2}\right)\right]^{T}, x=\left[x_{11}, x_{12}, x_{21}, x_{22}\right]^{T}$. Then, the system (81) can be given in the following form

$$
\ddot{y}=F(x)+G(x) u+H_{\tau}\left(x_{\tau}\right) \text {, }
$$

where

$$
\begin{aligned}
& F(x)=\left(\begin{array}{c}
x_{21}-0.3 \sin \left(x_{11} x_{12}\right)+x_{12}^{2} \\
x_{22}^{2}+x_{11}+x_{12}^{2}
\end{array}\right), \quad G(x)=\left(\begin{array}{cc}
\left(2+\cos \left(x_{11}\right)\right) & \left(1+x_{21}^{2}\right) \\
-1 & \left(1+x_{21}^{2}\right)
\end{array}\right), \\
& H_{\tau}\left(x_{\tau}\right)=\left(\begin{array}{c}
0.2 x_{11}\left(t-\tau_{1}(t)\right) \sin \left(x_{11}\left(t-\tau_{1}(t)\right)\right) \\
0.3 x_{21}\left(t-\tau_{2}(t)\right)
\end{array}\right) .
\end{aligned}
$$

Note that $N_{i}\left(v_{i}\right)$ for $i=1,2$ are the considered input nonlinearities. $v_{i}$, for $i=1,2$, are the control inputs, and $u_{i}$, for $i=1,2$, are the outputs of actuators (i.e. the outputs of dead-zones or backlash).

The control objective consists in allowing the system outputs $y_{1}$ and $y_{2}$ to respectively track the sinusoidal desired trajectories $y_{d 1}=\sin (t)$ and $y_{d 2}=\sin (t)$.

The fuzzy system $\theta_{1}^{T} \psi_{1}\left(\bar{z}_{1}\right)$ has the vector $\left[x^{T}, v_{2}\right]^{T}$ as input, while the fuzzy systems $\theta_{2}^{T} \psi_{2}\left(\bar{z}_{2}\right), W_{1}^{T} \varphi_{1}(x)$ and $W_{2}^{T} \varphi_{2}(x)$ have the state vector $x$ as input. For each variable of the entries of these fuzzy systems, as in [4], we define three (one triangular and two trapezoidal) membership functions uniformly distributed on the intervals [-2,2] for $x_{11}, x_{12}, x_{21}$, and $x_{22}$, and [-25,25] for $v_{2}$. The design parameters are chosen as follows: $\gamma_{01}=\gamma_{02}=1, \gamma_{11}=300, \gamma_{12}=150, \gamma_{21}=\gamma_{22}=1, \gamma_{31}=\gamma_{32}=250, \sigma_{1}=\sigma_{2}=0$, $001, \delta_{1}=\delta_{2}=0,005, \lambda_{1}=\lambda_{2}=2, k_{11}=k_{12}=2$. The initial conditions are selected as: $x\left(\begin{array}{ll}0 \\ 0\end{array}=\left[\begin{array}{llll}0.5 & 0 & 0.5 & 0\end{array}\right], k_{01}(0)=\right.$ $k_{02}(0)=0, k_{21}(\mathbf{0})=k_{22}(0)=0, \theta_{1}(0)=\underline{0}, \theta_{2}(\mathbf{0})=\underline{0}, W_{1}(\mathbf{0})=\underline{0}$ and $W_{2}(0)=\underline{0}$. The time-delays are chosen as follows: $\tau_{1}(t)=0.2(1+\sin (t)), \tau_{2}(t)=1-0.5 \cos (t)$.

(a) Simulation with dead-zone nonlinearity in the input We also consider the dead-zone model given in (79). Its parameters are selected as follows: $b_{r 1}=b_{r 2}=3$, $b_{l 1}=b_{l 2}=-2.25, m_{1}=m_{2}=2$. Fig. 3 shows the second fuzzy adaptive controller performs well in spite of the input nonlinearities, uncertainties and time-varying delays. In fact, Fig. 3(a) and (b) illustrate the boundedness and convergence of the tracking errors for both subsystems. The boundedness of the corresponding control signals is well illustrated in Fig. 3(c) and (d). So, clearly, these simulation results verify our theoretical findings. 

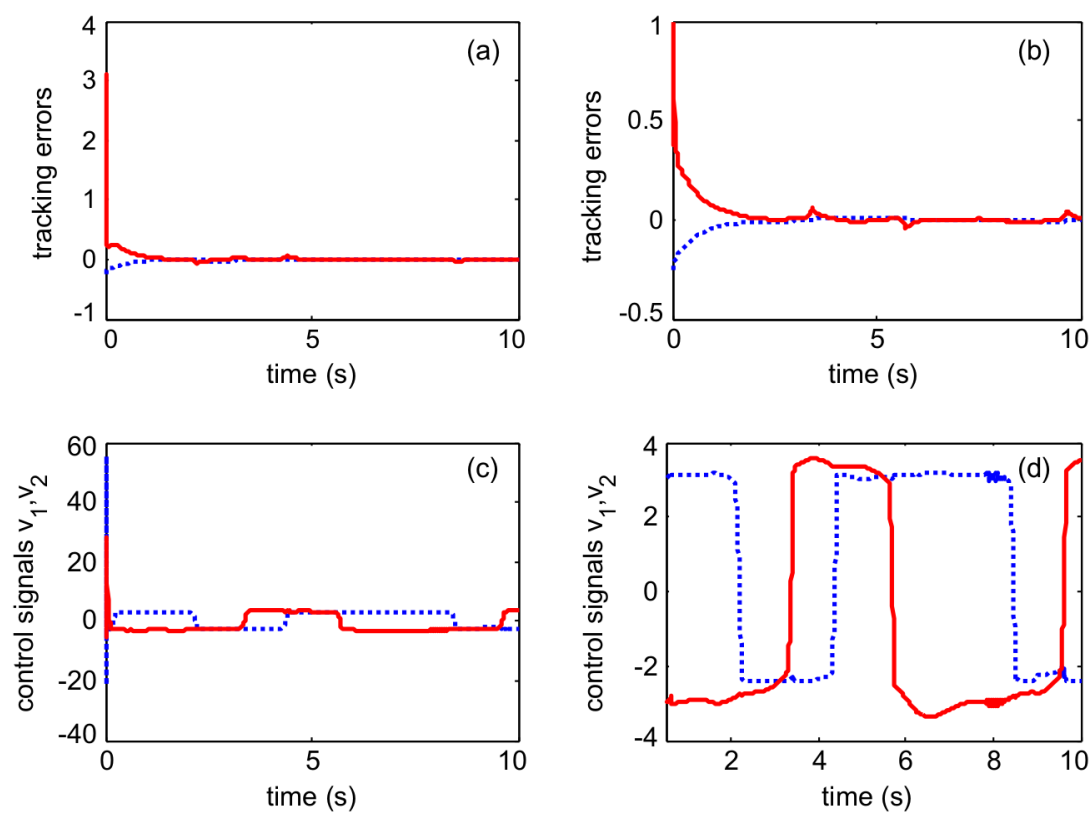

Fig. 3. Simulation results with dead-zone (Example 2). (a) Tracking errors : $e_{1}$ (dotted line) and $\dot{e}_{1}$ (solid line). (b) Tracking errors : $e_{2}$ (dotted line) and $\dot{e}_{2}$ (solid line). (c) Control input signals: $v_{1}$ (dotted line) and $v_{2}$ (solid line). (d) Control input signals for $t \in[0.5,10]: v_{1}$ (dotted line) and $v_{2}$ (solid line).
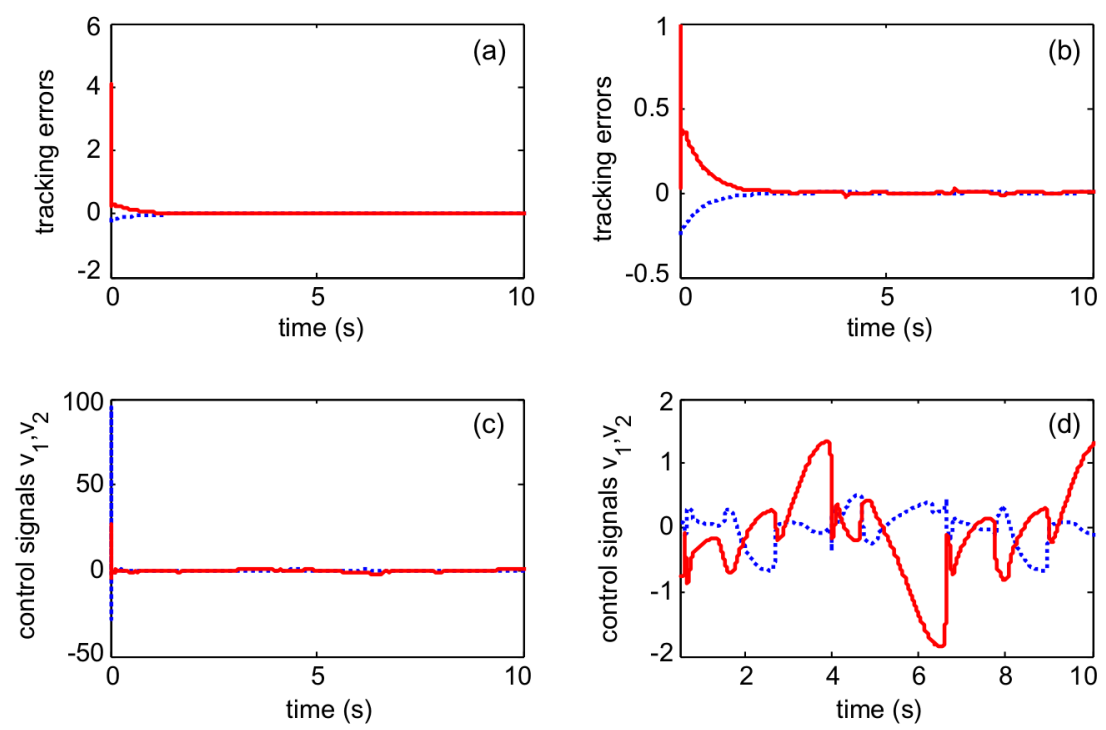

Fig. 4. Simulation results with backlash (Example 2). (a) Tracking errors: $e_{1}$ (dotted line) and $\dot{e}_{1}$ (solid line). (b) Tracking errors : $e_{2}$ (dotted line) and $\dot{e}_{2}$ (solid line). (c) Control input signals: $v_{1}$ (dotted line) and $v_{2}$ (solid line). (d) Control input signals for $t \in[0.5,10]: v_{1}$ (dotted line) and $v_{2}$ (solid line).

(b) Simulation with backlash hysteresis in the input

We consider again the backlash model given in (80). Its parameters are chosen as follows: $a_{1}=a_{2}=0.5, c_{1}=c_{2}=3.1635$, and $h_{1}=h_{2}=0.345$. Fig. 4 shows the simulation results of the second fuzzy adaptive controller by taking into account the backlash hysteresis.

\section{Conclusion}

Two fuzzy adaptive control algorithms for multivariable unknown nonlinear systems with both unknown actuator nonlinearities and unknown control direction have been presented. The first one has been designed for system without 
time-delays, while the second one has been designed for systems with time-varying delays. Two design features are worth to be mentioned. Firstly, the involved adaptive compensation of the dead-zone and the backlash hysteresis requires neither the knowledge of their parameters nor the construction of their inverses. Secondly, the knowledge of the control direction is not required thanks to the genuine concept of Nussbaum function. Of fundamental interest, it has been shown that the underlying control systems are stable and that the involved tracking errors converge to the origin. The effectiveness of the proposed controllers is particularly emphasized throughout a set of simulation results.

\section{References}

[1] N.J. Ahmad, F. Khorrami, Adaptive control of systems with backlash hysteresis at the input, in: Proceedings of American Control Conference, 1999, pp. 3018-3022.

[2] E.-W. Bai, Adaptive dead-zone inverse for possibly nonlinear control systems, in: Adaptive Control of Nonsmooth Dynamic Systems, Springer, New work, 2001, pp. 31-47.

[3] A. Boulkroune, M. Tadjine, M. M'Saad, M. Farza, General adaptive observer-based fuzzy control of uncertain nonaffine systems, Archives of Control Sciences 16 (LII 4) (2006) 363-390.

[4] A. Boulkroune, M. Tadjine, M. M'Saad, M. Farza, How to design a fuzzy adaptive controller based on observers for uncertain affine nonlinear systems, Fuzzy Sets and Systems 159 (2008) 926-948.

[5] A. Boulkroune, M. M'Saad, M. Tadjine, M. Farza, Adaptive fuzzy control of MIMO nonlinear systems with unknown hysteresis and control gain matrix sign, in: Proceedings of 16th IEEE Mediterranean Conference on Control and Automation, Ajaccio, France, June 2008, pp. $380-385$.

[6] A. Boulkroune, M. M'Saad, M. Tadjine, M. Farza, Adaptive fuzzy control for MIMO nonlinear systems with unknown dead-zone, in: Proceedings of Fourth International IEEE Conference on Intelligent Systems, Varna, Bulgaria, September 2008, pp. 450-455.

[7] Y.C. Chang, Robust tracking control for nonlinear MIMO systems via fuzzy approaches, Automatica 36 (2000) 1535-1545.

[8] H. Chekireb, M. Tadjine, D. Bouchaffra, Direct adaptive fuzzy control of nonlinear system class with applications, Control and Intelligent Systems 31 (2) (2003) 1-11.

[9] B. Chen, X.P. Liu, S.C. Tong, Adaptive fuzzy output tracking control of MIMO nonlinear uncertain systems, IEEE Transactions on Fuzzy Systems 15 (2) (2007) 287-300.

[10] B. Chen, X.P. Liu, K.F. Liu, P. Shi, C. Lin, Direct adaptive fuzzy control for nonlinear systems with time-varying delays, Information Sciences 180 (2010) 776-792.

[11] C.-S. Chen, Dynamic structure adaptive neural fuzzy control for MIMO uncertain nonlinear systems, Information Sciences 179 (2009) $2676-2688$.

[12] C.Y. Chen, T.H.S. Li, Y.C. Yeh, EP-based kinematic control and adaptive fuzzy sliding-mode dynamic control for wheeled mobile robots, Information Sciences 179 (2009) 180-195.

[13] J. Chen, A. Behal, D.M. Dawson, Adaptive output feedback control for a class of MIMO nonlinear systems, in: Proceedings of the American Control Conference, Minneapolis, MN, June 2006, pp. 5300-5305.

[14] H.Y. Cho, E.W. Bai, Convergence results for an adaptive dead zone inverse, International Journal of Adaptive Control and Signal Processing 12 (5) (1998) 451-466.

[15] R.R. Costa, Li. Hsu, A.K. Imai, P. Kokotovic, Lyapunov-based adaptive control of MIMO systems, Automatica 39 (7) (2003) $1251-1257$.

[16] N. Essounbouli, A. Hamzaoui, J. Zaytoon, An improved robust adaptive fuzzy controller for MIMO systems, Control and Intelligent Systems 34 (1) (2006) 12-21.

[17] S.S. Ge, J. Wang, Robust adaptive neural control for a class of perturbed strict feedback nonlinear systems, IEEE Transactions on Neural Networks 13 (6) (2002) 1409-1419.

[18] S.S. Ge, F. Hong, T.H. Lee, Adaptive neural network control of nonlinear systems with unknown time delays, IEEE Transactions on Automatic Control 48 (11) (2003) 2004-2010.

[19] S.S. Ge, F. Hong, T.H. Lee, Adaptive neural control of nonlinear time-delay system with unknown virtual control coefficients, IEEE Transactions on Systems, Man, and Cybernetics-PartB: Cybernetics 34 (1) (2004) 499-516.

[20] S.S. Ge, K.P. Tee, Approximation-based control of nonlinear MIMO time-delay systems, Automatica 43 (2007) $31-43$.

[21] N. Golea, A. Golea, K. Benmahammed, Stable indirect fuzzy adaptive control, Fuzzy Sets and Systems 137 (2003) $353-366$.

[22] K. Gu, V.L. Kharitonov, J. Chen, Stability of Time-Delay Systems, Birkhauser, Boston, 2003.

[23] L. Hsu, R.R. Costa, F. Lizarralde, Luapunov/passivity-based adaptive control of relative degree two MIMO systems with an application to visual servoing, IEEE Transactions on Automatic Control 52 (2) (2007) 364-371.

[24] C. Hua, F. Li, X. Guan, Observer-based adaptive control for uncertain time-delay systems, Information Sciences 176 (2) (2006) $201-214$.

[25] J.O. Jang, A dead-zone compensator of a DC motor system using fuzzy logic control, IEEE Transactions on Systems, Man, and Cybernetics - Part C 31 (1) (2001) 42-47.

[26] V.L. Kharitonov, D. Melchor-Aguilar, Lyapunov-Krasovskii functionals for additional dynamics, International Journal of Robust Nonlinear Control 13 (2003) 793-804.

[27] J.-H. Kim, J.-H. Park, S.-W. Lee, E.K.P. Chong, A two-layered fuzzy logic controller for systems with dead-zones, IEEE Transactions on Industrial Electronics 41 (2) (1994) 155-161.

[28] V.B. Kolmanovskii, J. Richard, Stability of some linear systems with delays, IEEE Transactions on Automatic Control 44 (5) (1999) 984-989.

[29] M.A. Krasnoskl'skii, A.V. Pokrovskii, Systems with Hysteresis, Nauka, Moscow, Russia, 1983.

[30] S. Labiod, M.S. Boucherit, T.M. Guerra, Adaptive fuzzy control of a class of MIMO nonlinear systems, Fuzzy Sets and Systems 151 (2005) 59-77.

[31] F.L. Lewis, W.K. Tim, L.-Z. Wang, Z.-X. Li, Dead-zone compensation in motion control systems using adaptive fuzzy logic control, IEEE Transactions on Control Systems Technology 7 (6) (1999) 731-741.

[32] H.-X. Li, S.C. Tong, A hybrid adaptive fuzzy control for a class of nonlinear MIMO systems, IEEE Transactions on Fuzzy Systems 11 (1) (2003) 24-34.

[33] Y.-J. Liu, W. Wang, Adaptive fuzzy control for a class of uncertain nonaffine nonlinear systems, Information Sciences 177 (18) (2007) 3901-3917.

[34] J.W. Macki, P. Nistri, P. Zecca, Mathematical models for hysteresis, SIAM Review 35 (1993) 94-123.

[35] I.D. Mayergoyz, The Preisach Model for Hysteresis, Springer-Verlag, Berlin, Germany, 1991.

[36] K.S. Narendra, M.A. Annaswamy, A new adaptive law for robust adaptive control without persistent excitation, IEEE Transactions on Automatic Control 32 (1987) 134-145.

[37] R.D. Nussbaum, Some remarks on the conjecture in parameter adaptive control, Systems \& Control Letters 1 (3) (1983) $243-246$.

[38] R. Ordonez, K.M. Passino, Stable multi-input multi-output adaptive fuzzy/neural control, IEEE Transactions on Fuzzy Systems 7 (3) (1999) 345-353.

[39] R.R. Selmic, F.L. Lewis, Dead-zone compensation in motion control systems using neural networks, IEEE Transactions on Automatic Control 45 (4) (2000) 602-613.

[40] J.E. Slotine, W. Li, Applied Nonlinear Control, Prentice-Hall, Englewood Cliffs, NJ, 1991.

[41] G. Strang, Linear Algebra and its applications, second ed., Academic press, Inc., New Work, 1980.

[42] C.-Y. Su, Y. Stepanenko, J. Svoboda, T.P. Leung, Robust adaptive control of a class of nonlinear systems with unknown backlash-like hysteresis, IEEE Transactions on Automatic Control 45 (12) (2000) 2427-2432.

[43] C.-Y. Su, M. Oya, H. Hong, Stable adaptive fuzzy control of nonlinear systems preceded by unknown backlash-like hysteresis, IEEE Transactions on Fuzzy Systems 11 (1) (2003) 1-8. 
[44] X. Sun, W. Zhang, Y. Jin, Stable adaptive control of backlash nonlinear systems with bounded disturbance, in: Proceedings of 31th Conference on Decision Control, 1992, pp. 274-275.

[45] Y. Sun, J. Hsieh, H. Yang, On the stability of uncertain systems with multiple time-varying delays, IEEE Transactions on Automatic Control 42 (1) (1997) $101-105$.

[46] G. Tao, P.V. Kokotovic, Adaptive sliding control of plants with unknown dead-zone, IEEE Transactions on Automatic Control 39 (1) (1994) 59-68.

[47] G. Tao, P.V. Kokotovic, Discrete-time adaptive control of systems with unknown dead-zone, International Journal of Control 61 (1)(1995) 1-17.

[48] G. Tao, P.V. Kokotovic, Adaptive control of plants with unknown hysteresis, IEEE Transactions on Automatic Control 40 (1995) $200-212$.

[49] G. Tao, P.V. Kokotovic, Continuous-time adaptive control of systems with unknown backlash, IEEE Transactions on Automatic Control 40 (1995) $1083-$ 1087.

[50] S.C. Tong, J. Tang, T. Wang, Fuzzy adaptive control of multivariable nonlinear systems, Fuzzy Sets and Systems 111 (2) (2000) $153-167$.

[51] S.C. Tong, H.X. Li, Fuzzy adaptive sliding model control for MIMO nonlinear systems, IEEE Transactions on Fuzzy Systems 11 (3) (2003) 354-360,

[52] S.C. Tong, B. Chen, Y. Wang, Fuzzy adaptive output feedback control for MIMO nonlinear systems, Fuzzy Sets and Systems 156 (2) (2005) 285-299.

[53] S.C. Tong Y. Li, P. Shi, Fuzzy adaptive backstepping robust control for SISO nonlinear system with dynamic uncertainties, Information Sciences 179 (2009) 1319-1332.

[54] S.C. Tong, X.L. He, Y.M. Li, Direct adaptive fuzzy backstepping robust control for single input and single output uncertain nonlinear systems using small-gain approach, Information Sciences 180 (9) (2010) 1738-1758.

[55] M. Wang, B. Chen, K. Liu, X. Liu, S. Zhang, Adaptive fuzzy tracking control of nonlinear time-delay systems with unknown virtual control coefficients, Information sciences 178 (2008) 4326-4340.

[56] L.X. Wang, Adaptive Fuzzy Systems and Control: Design and Stability Analysis, Prentice-Hall, Englewood Cliffs, NJ, 1994

[57] X.-S. Wang, H. Hong, C.Y. Su, Model reference adaptive control of continuous-time systems with an unknown dead-zone, IEE Proceedings of Control Theory Applications 150 (3) (2003) 261-266.

[58] X.-S. Wang, C.-Y. Su, H. Hong, Robust adaptive control of a class of nonlinear systems with unknown dead-zone, Automatica 40 (3) (2004) $407-413$.

[59] C. Wen, J. Zhou, Decentralized adaptive stabilization in the presence of unknown backlash-like hysteresis, Automatica 43 (2007) $426-440$.

[60] Y. Yang, C. Zhou, Robust adaptive fuzzy tracking control for a class of perturbed strict-feedback nonlinear systems via small-gain approach, Information Sciences $170(2-4)(2005)$ 211-234.

[61] X.D. Ye, Adaptive nonlinear output-feedback control with unknown high-frequency gain sign, IEEE Transactions on Automatic Control 46 (1) (2001) $112-115$.

[62] T.P. Zhang, S.S. Ge, Adaptive neural control of MIMO nonlinear state time-varying delay systems with unknown dead-zones and gain signs, Automatica 43 (6) (2007) 1021-1033.

[63] T.P. Zhang, Y. YI, Adaptive fuzzy control for a class of MIMO nonlinear systems with unknown dead-zones, Acta Automatica Sinica 33 (1) (2007) 96-99.

[64] X.T. Zhang, D.M. Dawson, M.S. de Queiroz, B. Xian, Adaptive control for a class of MIMO nonlinear systems with non-symmetric input matrix, in: Proceedings of the IEEE International Conference on Control Applications, Taipei, Taiwan, September 2004, pp. 1324-1329.

[65] J. Zhou, C. Wen, Y. Zhang, Adaptive backstepping control of a class of uncertain nonlinear systems with unknown backlash-like hysteresis, IEEE Transactions on Automatic Control 49 (10) (2004) 1751-1757.

[66] J. Zhou, C. Wen, Y. Zhang, Adaptive output control of nonlinear systems with uncertain dead-zone nonlinearity, IEEE Transactions on Automatic Control 51 (3) (2006) 504-511.

[67] J. Zhou, C. Zhang, C. Wen, Robust adaptive output control of uncertain nonlinear plants with unknown backlash nonlinearity, IEEE Transactions on Automatic Control 52 (3) (2007) 503-509. 